In Diagnosis and Control of Diseases of Fish and Shellfish. Chap. 9. pp 223-261

2017

Eds Brian Austin, Aweeda Newaj-Fyzul

ISBN 9781119152101

https://doi.org/10.1002/9781119152125.ch9

https://archimer.ifremer.fr/doc/00748/86031/

\title{
Use of Medicinal Plants in Aquaculture
}

\author{
Reverter Miriam 1, Tapissier-Bontemps Nathalie ${ }^{1}$, Sasal Pierre ${ }^{1}$, Saulnier Denis ${ }^{2}$
}

${ }^{1}$ CRIOBE, Paris Sciences et Lettres (PSL), University of Perpignan Via Domitia, Perpignan, France

2 Ifremer, Tahiti, French Polynesi, France.

\begin{abstract}
:
Plants have been reported to produce various effects such as antistress, growth promotion, appetite stimulation, immunostimulation, aphrodisiac and to have antipathogen properties in fish and shrimp aquaculture due to their varied active principles such as alkaloids, terpenoids, tannins, saponins and flavonoids. To date, most scientific studies on the use of medicinal plants in aquaculture have focused on identification of biological activity rather than natural product determination. The plant species that have displayed the highest potential for use in aquaculture are garlic (Allium sativum), pomegranate (Punica granatum), bermuda grass (Cynodon dactylon), Indian ginseng (Whitania somnifera) and ginger (Zingiber officinale). Algae are considered to be a rich source of original bioactive molecules which display multiple bioactivities. In aquaculture, several recent studies have showed the potential of algae for the treatment of pathogens or to improve fish fitness.
\end{abstract}

Keywords : aquaculture disease management, bioactive algae, biological activity, fish farmers, medical plants, shrimp aquaculture 


\section{Introduction}

For thousands of years, civilizations throughout the world have used medicinal plants to treat a variety of diseases. The oldest written evidence of medicinal plant usage was found on a Sumerian clay slab, approximately 5000 years old (Petrovska, 2012). Nowadays, traditional medicinal plants continue to be the primary source of healthcare in many developing countries and rural regions (Calixto, 2005). Ethnobotanical studies have proved to be very useful in identifying bioactive plants, and numerous research studies have been conducted on the biological activities and chemical composition of ethnomedicinal plants (Ayyanar et al., 2011; Banskota et al., 2003). Interest in medicinal plants for human and veterinary health has been fuelled by the countless side effects and rising costs of prescription drugs (Hoareau and Da-Silva, 1999). Plants also possess a complex chemical composition which displays varied biological activities, making plants suitable for the treatment of multifactorial diseases, and makes plants a suitable alternative to antibiotics with little risk for development of resistance (Gostner et al., 2012; Srivastava et al., 2014).

Aquaculture is the fastest growing animal food-producing sector, with an average annual increase of $6.2 \%$ per year in the period 2000-2012 (FAO, 2014). However, aquaculture growth is often linked to culture intensification, leading to overcrowding and poor water quality, facilitating the spread of pathogens and increasing disease outbreaks and mortality (Bondad-Reantaso et al., 2005). In order to avoid economic losses related to sanitary shortcomings, veterinary drugs are commonly used in aquaculture to prevent and treat disease outbreaks (Rico et al., 2013).

The intensive use of synthetic drugs presents numerous disadvantages, for both the environment and health. Intensive use of antibiotics has resulted in accumulation in muscle of commercialized animals (Cabello et al., 2006; Romero-Ormazabal et al., 2012) and the development of resistant bacteria strains (Miranda and Zemelman, 2002; Seyfried et al., 2010). Also, the use of antiparasitic drugs like trichlorfon or praziquantel in bath treatments is hazardous for animals and the environment and can also result in the development of resistance (Forwood et al., 2013; Umeda et al., 2006). Vaccines, considered to be the most effective method to prevent disease outbreaks in aquaculture, are too expensive for widespread use

Diagnosis and Control of Diseases of Fish and Shellfish, First Edition. Edited by Brian Austin and Aweeda Newaj-Fyzul. ๑ 2017 John Wiley \& Sons Ltd. Published 2017 by John Wiley \& Sons Ltd. 
by fish producers and since it is extremely difficult to develop multiple strain vaccines, most vaccines are only effective against one type of pathogen (Pasnik et al., 2005; Sakai, 1999).

Considering the numerous disadvantages of synthetic drugs, there is an increasing need for the development of alternative strategies in aquaculture disease management. Moreover, disease outbreaks in aquaculture are often associated with animal fitness and health, most pathogens being opportunistic and taking advantage of immunocompromised or stressed fish. Thus, alternative solutions should maximize fish immunity and fitness as a strategy to face pathogen infections (Ashley et al., 2007; Davis et al., 2002; Iguchi et al., 2003). Medicinal plants can therefore provide a cheaper and more sustainable alternative to chemotherapy in aquaculture, since they have been reported to display numerous bioactivity such as antistress, immunostimulant and antiparasitic (bacterial, fungus, virus and ectoparasites) effects (Reverter et al., 2014).

\section{Medicinal Plants in Aquaculture}

Although interest in the use of medicinal plants and plant extracts in aquaculture has exploded recently, medicinal plants have long been used by rural fish farmers. Caruso et al. (2013) found that $46 \%$ of the fish farmers surveyed in West Java (Indonesia) used plants in their farms, most of which were also traditionally used in the human pharmacopoeia. In most cases, fresh plants were directly introduced into the rearing water and used to improve water quality, reduce fish stress, increase fish resistance to pathogens and treat fish diseases. This study showed that fish farmers used plants and doses depending on their personal experience and ethnic origin.

Numerous scientific studies endorse the use of medicinal plants in aquaculture (Bhuvansewari and Balasundaram, 2006; Harikrishnan et al., 2011a). Plants have been reported to produce various effects such as antistress, growth promotion, appetite stimulation, immunostimulation, aphrodisiac and to have antipathogen properties in fish and shrimp aquaculture due to their varied active principles such as alkaloids, terpenoids, tannins, saponins and flavonoids (Chakraborty and Hancz, 2011; Citarasu, 2010).

\section{Biological Activity of Medicinal Plants in Aquaculture}

Several plant extracts are reported to stimulate appetite and promote weight gain when administered to cultured fish (Pavaraj et al., 2011; Takaoka et al., 2011). For example, grouper (Epinephelus tauvina) fed with a supplemented diet of a mixture of methanolic herb extracts (Cynodon dactylon, Piper longum, Phyllanthus niruri, Tridax procumbens and Zingiber officinalis) displayed $41 \%$ higher weight than control fish (Punitha et al., 2008). Other studies have reported that administration of plant extracts improves digestibility and availability of nutrients, resulting in an increase in feed conversion and leading to higher protein synthesis (Putra et al., 2013; Talpur et al., 2013).

Numerous studies have shown that fish treated with medicinal plants presented enhanced immune parameters (Dügenci et al., 2003; Yuan et al., 2007). For example, Nile tilapia (Oreochromis niloticus) fed with a diet containing mistletoe (Viscum album coloratum) for a period of 80 days displayed higher lysozyme, respiratory burst, alternative complement and phagocytic activity, which resulted in $42 \%$ increased survivability when they were challenged with the bacterial pathogen Aeromonas hydrophila (Park and Choi, 2012). Studies on fish haematological parameters have shown that fish treated with plants displayed higher levels of 
erythrocytes, lymphocytes, monocytes and haemoglobin than control fish, indicating better fish fitness (Haghighi and Rohani, 2013; Harikrishnan et al., 2012a).

In vitro and in vivo studies have shown the potential of medicinal plants against a wide range of marine pathogens (bacteria, virus, fungus and ectoparasites) (Chitmanat et al., 2005; Direkbusarakom et al., 1996; Ji et al., 2012). Antibacterial properties of medicinal plants are by far the best studied biological activities, with abundant in vitro studies reporting antibacterial activity in numerous plants against both Gram-positive and Gram-negative marine bacteria (Castro et al., 2008; Roomiani et al., 2013). In vivo studies in Indian major carp (Labeo rohita) fed with diets enriched with prickly chaff flower (Achyrantes aspera, $0,2 \%$ ) and Indian ginseng (Withania somnifera, $0,5 \%$ ) showed a reduction in mortality of $41 \%$ and $49 \%$ respectively when fish were challenged with A. hydrophila (Sharma et al., 2010; Vasudeva-Rao et al., 2006).

Antiviral and antifungal activities of medicinal plants are also able to prevent high mortality rates in aquaculture. Balasubramanian et al. (2008a,2008b) showed that black tiger shrimp (Penaeus monodon) challenged with white spot syndrome virus (WSSV) while being treated with Bermuda grass (Cynodon dactylon) displayed no mortality and no signs of disease compared to $100 \%$ mortality observed in control groups. Several studies have shown in vitro antifungal activities of several plants like conidinium fruit (Cnidium monnieri), magnolia bark (Magnolia officinalis), aucklandia root (Aucklandia lappa) and common rue (Ruta graveolens) (Hashemi et al., 2012; Xue-Gang et al., 2013).

Medicinal plants also seem to be an effective alternative for treating ectoparasites. Several studies have shown antiparasitic activities of medicinal plants when added to water or administered orally (Fu et al., 2014; Huang et al., 2013; Yi et al., 2012). Methanol extract of bupleurum root (Radix bupleuri chinensis), aqueous and methanol extracts of cinnamon (Cinnamomum cassia), methanol extract of Chinese spice bush (Lindera aggregata) and methanol and ethyl acetate extracts of golden larch (Pseudolarix kaempferi) had $100 \%$ in vivo efficacy against monogenean Dactylogyrus intermedium in infected goldfish (Carassius auratus) (Ji et al., 2012; Wu et al., 2011). Harikrishnan et al. (2012c) showed 40\% mortality decrease and enhanced immunity in olive flounder (Paralichthys olivaceus) infected by the protozoan ciliate Miamiensis avidus when fish were fed with a diet supplemented with Suaeda maxima.

\section{Application of Medicinal Plants in Aquaculture}

Plants can be administered as a whole plant or parts (leaf, root, seed, fruit) and can either be used fresh or as prepared herbal extracts with different solvents (water, methanol, chloroform, ethyl acetate) (van Hai, 2015). Biological activity and chemical composition of plants and extracts can vary greatly depending on the part used and type of extract, and thus knowledge of the plant's bioactive compounds is required. In addition, seasonal variations in production of bioactive metabolites has also been observed in different plants and algae, so study of the plant's production of bioactive molecules is desirable (Chaves et al., 2013; Manilal et al., 2009).

To date, most scientific studies on the use of medicinal plants in aquaculture have focused on identification of biological activity rather than natural product determination (Balasubramanian et al., 2007; Cox et al., 2010; Ji et al., 2012). Exploratory studies on the potential of medicinal plants in aquaculture can therefore be misleading if the wrong part or wrong solvent is used. Also, suitable dosing is crucial to obtain the desired effects, since inappropriate doses can display toxic effects in fish (Ekanem et al., 2007; Kavitha et al., 2012; Sambasivam et al., 2003). For example, grouper (Epinephelus coioides) fed with a diet supplemented with $1 \%$ ethanol katuk extract (Sauropus androgynous) showed enhanced growth and improved food 
utilization, whereas at $2.5 \%$ and $5 \%$ of katuk extract they presented lower growth levels (Putra et al., 2013). Treatment length is another important parameter in medicinal plant application in aquaculture, since it directly influences treatment effectiveness. For example, Militz et al. (2013) showed that farmed barramundii (Lates calcarifer) fed with an enriched diet of garlic (Allium sativum) for 30 days displayed 70\% decreased Neobenedenia sp. infection success compared to control and short-term treatment (10 days).

Medicinal plants can be administered to fish and shellfish by injection (intramuscular and intraperitoneal), oral administration and through immersion or baths (Ji et al., 2012; Putra et al., 2013; Wu et al., 2010). Although intraperitoneal injection has proved to be the most rapid and efficient method of administration, it is expensive, laborious and stressful for fish, especially for very young specimens (Anderson, 1992; Yoshida et al., 1995). Baths are extensively used for the treatment of ectoparasites (Forwood et al., 2013; Whittington, 2012) but this method is also expensive and laborious and involves the release of exogenous molecules in the marine environment, and thus can present some undesired environmental side effects (Umeda et al., 2006). Hence, oral administration seems to be the most suitable for aquaculture, since medicinal plants can induce physiological changes in fish to improve their fitness and reinforce their resistance to pathogens.

Finally, determining natural plant products helps to better understand their mode of action. For example, chelerythrine and chelidonine are two benzo[c] phenanthridines with antimicrobial, antifungal and anti-inflammatory activities isolated from greater celandine (Chelidonium majus L.) (Malikova et al., 2006; Waltérova et al., 1995). Several studies have shown that chelerythrine exhibits high cytotoxic potency against a large number of cells, through multiple apoptosis-inducing pathways, indicating that a direct action on mitochondria may be involved in the eradication of parasites (Kaminskyy et al., 2008; Kemény-Beke et al., 2006; Slaninová et al., 2001). Other studies have shown that the mechanism of action of chelidonine against the monogenean ectoparasite Dactylogyrus intermedium may be through the mitochondrial cell death pathway activated via caspase-9 (Philchenkov et al., 2008). Therefore, it is of high importance to identify the bioactive molecules for the observed bioactivities and standardize extraction procedures depending on the molecules identified, application procedures and doses, duration of treatments and type of pathogen (Reverter et al., 2014).

It is important to state that modes of action and toxicity of many plants are still poorly understood, so more research is needed before they can be safely used in aquaculture. Many of the plant bioactive molecules can also be toxic or antinutritional in fish, decreasing efficient utilization of feed nutrients or causing intestinal dysfunction, so their effect on fish physiology and adequate dosing needs to be studied (Krogdahl et al., 2010). In order to fill in the gaps in current knowledge, a structured research plan should be followed, focused on, first, bioactivity, compounds and modes of actions of the plants, and second on the plant effect on the organism's physiology and its appropriate use. In Figure 9.1, we propose some directions for research on medicinal plant utilization in aquaculture. In vitro tests should be performed in order to determine plant bioactivity, together with bioassay-guided fractionation to identify and characterize the bioactive natural products. In vitro tests should also be carried out to test the cytotoxicity of plant extracts on fish cells. Then in vivo tests on model species should be performed, first to understand the plant effects on animal physiology and determine the best conditions for plant application (mode of application, plant part and preparation, dose and length of treatment), and second, to estimate plant efficiency in organism survival against different pathogenic infections. Haematology indicators such as haematocrit, haemoglobin, 
RESEARCH STEPS OF MEDICINAL PLANT USE IN AQUACULTURE
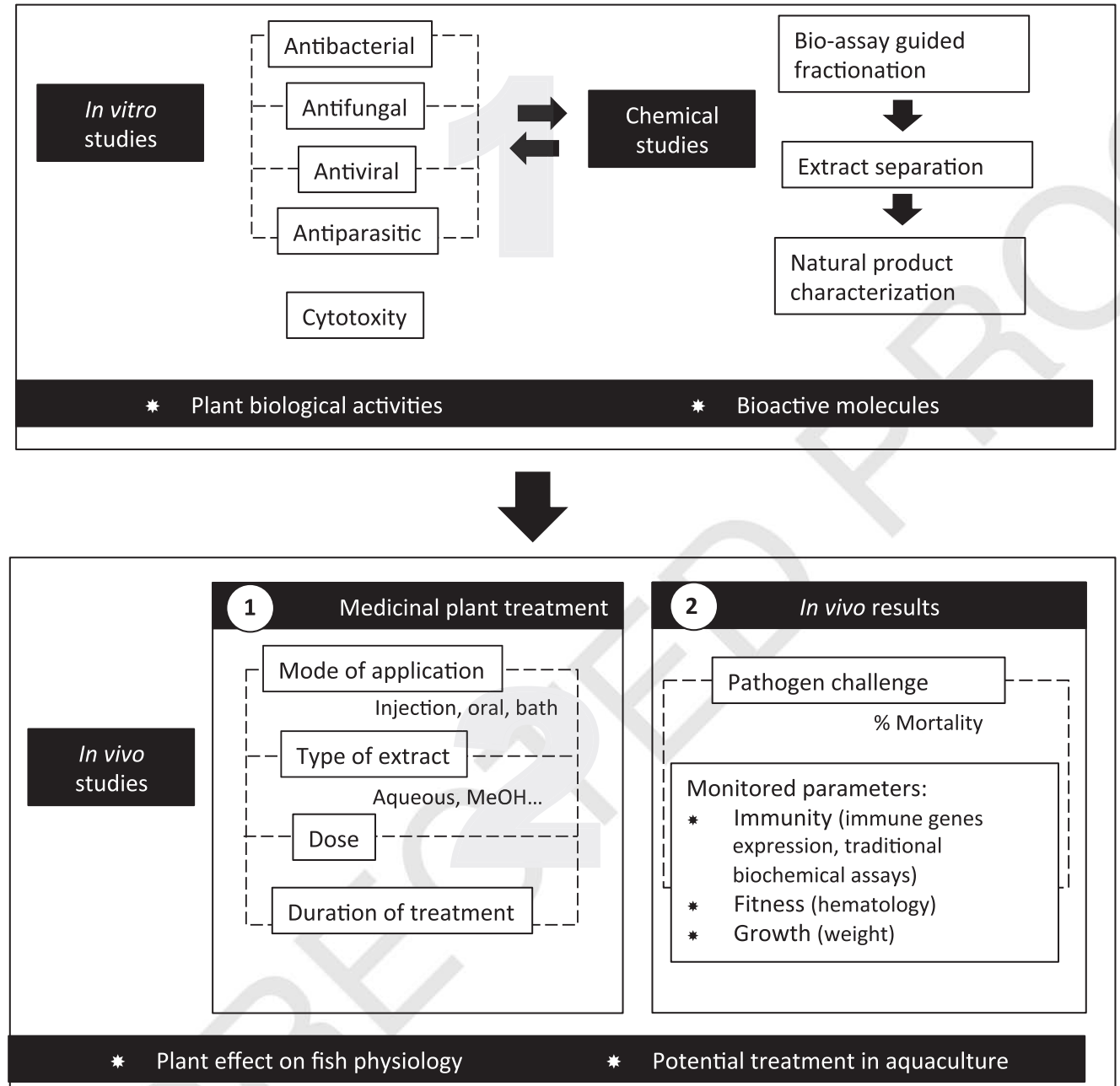

Figure 9.1 Research steps for medicinal plant utilization in aquaculture.

erythrocyte and lymphocyte levels can be used to determine fish fitness (Haghighi and Rohani, 2013; Kanani et al., 2014).

Fish immunity is often analysed to determine the immunomodulatory power of plants and this can be studied via classic biochemical approaches (lysozyme, phagocytic or respiratory burst activity) or by the study of immune gene expression (e.g. Lys, TNF-alpha, IL-1, IL-10 genes) (Chakrabarti et al., 2014; Harikrishnan et al., 2011a; Kumar et al., 2013).

\section{Analysis of Plants Used in Aquaculture}

An extensive literature review was performed on the published studies on plant use in aquaculture in order to identify the most common studied plants and their activities (Table 9.1). 
Diagnosis and Control of Diseases of Fish and Shellfish

Table 9.1 Plants, algae and mushrooms used or studied for potential application in aquaculture.

\begin{tabular}{|c|c|c|c|c|c|}
\hline Order & Family & Species & Activities & $\begin{array}{l}\text { Part } \\
\text { used }\end{array}$ & Reference \\
\hline Acorales & Acoraceae & Acorus calamus & AV & $\mathrm{R}$ & Sivasankar et al., 2015 \\
\hline Alismatales & Araceae & $\begin{array}{l}\text { Alocasia } \\
\text { macrorrhizos }\end{array}$ & NA & $\mathrm{L}$ & Caruso et al., 2013 \\
\hline Alismatales & Araceae & Colocasia esculenta & $\mathrm{AB}$ & $\mathrm{R}$ & Wei et al., 2010 \\
\hline Apiales & Apiaceae & $\begin{array}{l}\text { Angelica } \\
\text { membranaceus }\end{array}$ & $\mathrm{IM}$ & $\mathrm{R}$ & Jian and Wu, 2004 \\
\hline Apiales & Apiaceae & Angelica sinensis & IM & $\mathrm{R}$ & Wang et al., 2011 \\
\hline Apiales & Apiaceae & Angelica pubsecens & $\mathrm{AF}$ & $\mathrm{R}$ & Xue-Gang et al., 2013 \\
\hline Apiales & Apiaceae & Bupleurum chinense & AP & $\mathrm{W}$ & Wu et al., 2011 \\
\hline Apiales & Apiaceae & Centella asiatica & $\mathrm{AB}$ & $\mathrm{R}$ & $\begin{array}{l}\text { Rattanachaikunsopon and } \\
\text { Phumkhachorn, } 2010\end{array}$ \\
\hline Apiales & Apiaceae & Cnidium officinale & $\mathrm{AB}, \mathrm{GP}$ & $\mathrm{L}, \mathrm{Fr}$ & Takaoka et al., 2007b \\
\hline Apiales & Apiaceae & $\begin{array}{l}\text { Corandrium } \\
\text { sativum }\end{array}$ & $\mathrm{AB}$ & NA & Innocent et al., 2011 \\
\hline Apiales & Apiaceae & $\begin{array}{l}\text { Heracleum } \\
\text { lasiopetalum }\end{array}$ & $\mathrm{AB}$ & $\mathrm{R}$ & Pirbalouti et al., 2011 \\
\hline Apiales & Apiaceae & Radix peucedani & $\mathrm{AP}$ & W & Wu et al., 2011 \\
\hline Apiales & Araliaceae & Kalopanax pictus & $\mathrm{AP}$ & $\mathrm{S}$ & Harikrishnan et al., 2011e \\
\hline Apiales & Araliaceae & Panax ginseng & IM & $\mathrm{R}$ & Gooda, 2008 \\
\hline Apiales & Araliaceae & $\begin{array}{l}\text { Eleutherococcus } \\
\text { senticosus }\end{array}$ & $\begin{array}{l}\mathrm{AB} \\
\mathrm{IM}\end{array}$ & $\mathrm{R}$ & Won et al., 2008 \\
\hline Apiales & Araliaceae & $\begin{array}{l}\text { Panax } \\
\text { auinauefolium }\end{array}$ & GP & W & Abdel-Tawwab, 2012 \\
\hline Apiales & Umberlliferae & Carum carvi & GP & $\mathrm{L}$ & Abdel-Tawwab, 2011 \\
\hline Arecales & Arecaceae & Area catechu & AP & $\mathrm{L}$ & Caruso et al., 2013 \\
\hline Arecales & Arecaceae & Phoenix dactylifera & NA & $\mathrm{L}$ & Hoseinfar, 2015 \\
\hline Asparagales & Amaryllidaceae & Allium sativum & $\begin{array}{l}\mathrm{AB}, \\
\mathrm{AP} \\
\mathrm{IM}, \\
\mathrm{GP}\end{array}$ & W & $\begin{array}{l}\text { Aly et al., 2008; Nya and } \\
\text { Austin, 2009; Militz et al., } \\
\text { 2013; Sahu et al., } 2007\end{array}$ \\
\hline Asparagales & Amaryllidaceae & Allium tuberosum & $\begin{array}{l}\mathrm{AB} \\
\mathrm{GP}\end{array}$ & $\mathrm{L}$ & $\begin{array}{l}\text { Rattanachaikunsopon and } \\
\text { Phumkhachorn, 2009a }\end{array}$ \\
\hline Asparagales & Xanthorrhoeacea & Aloe vera & $\mathrm{AB}$ & W & $\begin{array}{l}\text { Alishani et al., } 2010 \text {; Kim } \\
\text { et al., } 1999\end{array}$ \\
\hline Asterales & Asteraceae & $\begin{array}{l}\text { Ageratum } \\
\text { conzyoides }\end{array}$ & NA & $\mathrm{L}$ & Caruso et al., 2013 \\
\hline Asterales & Asteraceae & Artemisia annua & AP & $\mathrm{L}$ & Martins et al., 2002 \\
\hline Asterales & Asteraceae & Artemisia argyi & $\begin{array}{l}\mathrm{AB} \\
\mathrm{AF} \\
\mathrm{AP}\end{array}$ & NA & Ekanem and Brisibe (2010) \\
\hline Asterales & Asteraceae & $\begin{array}{l}\text { Artemisia } \\
\text { capillaries }\end{array}$ & $\begin{array}{l}\mathrm{AB} \\
\mathrm{GP}\end{array}$ & $\mathrm{W}$ & Ji et al., 2007b \\
\hline
\end{tabular}


Table 9.1 (Continued)

\begin{tabular}{|c|c|c|c|c|c|}
\hline Order & Family & Species & Activities & $\begin{array}{l}\text { Part } \\
\text { used }\end{array}$ & Reference \\
\hline Asterales & Asteraceae & Artemisia cina & $\begin{array}{l}\mathrm{AB} \\
\mathrm{GP}\end{array}$ & W & Abdelhadi et al., 2010 \\
\hline Asterales & Asteraceae & Artemisia vulgaris & $\begin{array}{l}\mathrm{AB} \\
\mathrm{AV} \\
\mathrm{AP}\end{array}$ & NA & $\begin{array}{l}\text { El-Deen and Mohamed, } \\
2009\end{array}$ \\
\hline Asterales & Asteraceae & Aucklandia lappa & AV & NA & Xue-Gang et al., 2013 \\
\hline Asterales & Asteraceae & $\begin{array}{l}\text { Austroeupatorium } \\
\text { inulifolium }\end{array}$ & $\mathrm{NA}$ & NA & Caruso et al., 2013 \\
\hline Asterales & Asteraceae & $\begin{array}{l}\text { Chamaemelum } \\
\text { nobile }\end{array}$ & $\mathrm{AB}$ & NA & Syahidah et al., 2015 \\
\hline Asterales & Asteraceae & $\begin{array}{l}\text { Chrysanthemum } \\
\text { cinerariaefolium }\end{array}$ & $\begin{array}{l}\text { AP, } \\
\text { IM, } \\
\text { GP }\end{array}$ & NA & Harikrishnan et al., 2010d \\
\hline Asterales & Asteraceae & Cosmos caudatus & $\mathrm{AB}$ & NA & $\begin{array}{l}\text { Caruso et al., 2013, } \\
\text { Syahidah et al., } 2015\end{array}$ \\
\hline Asterales & Asteraceae & Echinacea purpura & $\begin{array}{l}\mathrm{AB} \\
\mathrm{AP}\end{array}$ & $\mathrm{L}, \mathrm{F}$ & $\begin{array}{l}\text { Aly and Mohamed, 2010; } \\
\text { Caruana et al., } 2012\end{array}$ \\
\hline Asterales & Asteraceae & Eclipta alba & $\begin{array}{l}\mathrm{AB} \\
\mathrm{GP}\end{array}$ & $\mathrm{L}, \mathrm{S}$ & Christybapita et al., 2007 \\
\hline Asterales & Asteraceae & $\begin{array}{l}\text { Erechtites } \\
\text { valerianifolia }\end{array}$ & NA & NA & Caruso et al., 2013 \\
\hline Asterales & Asteraceae & $\begin{array}{l}\text { Eupatorium } \\
\text { fortunei }\end{array}$ & $\mathrm{AP}$ & $\mathrm{S}$ & Huang et al., 2013 \\
\hline Asterales & Asteraceae & Gynura procumbens & NA & NA & Caruso et al., 2013 \\
\hline Asterales & Asteraceae & Lactuca indica & $\begin{array}{l}\mathrm{AB} \\
\mathrm{IM}\end{array}$ & NA & Harikrishnan et al., 2011c \\
\hline Asterales & Asteraceae & $\begin{array}{l}\text { Matricaria } \\
\text { chamomilla }\end{array}$ & $\begin{array}{l}\mathrm{AB} \\
\mathrm{GP}\end{array}$ & NA & Abdelhadi et al., 2010 \\
\hline Asterales & Asteraceae & Mikania scandens & NA & NA & Caruso et al., 2013 \\
\hline Asterales & Asteraceae & $\begin{array}{l}\text { Siegesbeckia } \\
\text { glabrescens }\end{array}$ & $\begin{array}{l}\mathrm{AB} \\
\mathrm{IM}\end{array}$ & W & Harikrishnan et al., 2012b \\
\hline Asterales & Asteraceae & $\begin{array}{l}\text { Spagneticola } \\
\text { calendulacea }\end{array}$ & $\mathrm{AB}$ & NA & $\begin{array}{l}\text { Direkbysarakom et al., } \\
2004\end{array}$ \\
\hline Asterales & Asteraceae & Tagetes erecta & $\begin{array}{l}\mathrm{AB} \\
\mathrm{AV}\end{array}$ & $\mathrm{L}$ & Caruso et al., 2013 \\
\hline Asterales & Asteraceae & Tithonia diversifolia & NA & NA & Caruso et al., 2013 \\
\hline Asterales & Asteraceae & Tridax procumbens & $\begin{array}{l}\mathrm{AV} \\
\mathrm{GP}\end{array}$ & W & Sivasankar et al., 2015 \\
\hline Asterales & Asteraceae & $\begin{array}{l}\text { Vernonia } \\
\text { amygdaluna }\end{array}$ & $\mathrm{AF}$ & NA & Syahidah et al., 2015 \\
\hline Brassicales & Caricaceae & Carica papaya & $\begin{array}{l}\mathrm{AP} \\
\mathrm{GP}\end{array}$ & NA & Ekanem et al., 2004 \\
\hline Brassicales & Moringaceae & Moringa oleifera & NA & W & Caruso et al., 2013 \\
\hline
\end{tabular}


Diagnosis and Control of Diseases of Fish and Shellfish

Table 9.1 (Continued)

\begin{tabular}{|c|c|c|c|c|c|}
\hline Order & Family & Species & Activities & $\begin{array}{l}\text { Part } \\
\text { used }\end{array}$ & Reference \\
\hline Caryophyllales & Amaranthaceae & Acyranthes aspera & $\begin{array}{l}\mathrm{AB} \\
\mathrm{IM}\end{array}$ & NA & Vasudeva-Rao et al., 2006 \\
\hline Caryophyllales & Amaranthaceae & Alteranthera sessilis & GP & $\begin{array}{l}\mathrm{L}, \mathrm{S} \\
\text { Fr }\end{array}$ & Radhakrishnan et al., 2014 \\
\hline Caryophyllales & Amaranthaceae & Kochia scoparia & AP & NA & Lu et al., 2012 \\
\hline Caryophyllales & Amaranthaceae & Suaeda maritima & $\begin{array}{l}\text { AP, } \\
\text { IM }\end{array}$ & NA & Harikrishnan et al., 2011c \\
\hline Caryophyllales & Cactaceae & Opuntia stricta & NA & $\mathrm{L}, \mathrm{F}$ & Khem, 2015 \\
\hline Caryophyllales & Caryophyllaceae & Stellaria aquatica & $\begin{array}{l}\mathrm{AB} \\
\mathrm{AV}\end{array}$ & NA & $\begin{array}{l}\text { Shangliang et al., } 1990 \\
\text { (siva) }\end{array}$ \\
\hline Caryophyllales & Molluginaceae & $\begin{array}{l}\text { Glinus } \\
\text { oppositifolius }\end{array}$ & AV & Fr & Sivasankar et al., 2015 \\
\hline Caryophyllales & Plumbaginaceae & $\begin{array}{l}\text { Aegialitis } \\
\text { rotundifolia }\end{array}$ & $\mathrm{AB}$ & $\mathrm{L}, \mathrm{W}$ & Choudhury et al., 2005 \\
\hline Caryophyllales & Polygonaceae & $\begin{array}{l}\text { Polyonum } \\
\text { hydropipper }\end{array}$ & $\begin{array}{l}\mathrm{AB} \\
\mathrm{AV}\end{array}$ & B & $\begin{array}{l}\text { Direkbysarakom et al., } \\
2004\end{array}$ \\
\hline Caryophyllales & Polygonaceae & Rheum officinale & $\mathrm{AB}$ & NA & Xie et al., 2008 \\
\hline Caryophyllales & Polygonaceae & Rumex obtusifolius & $\mathrm{AP}$ & $\mathrm{L}$ & Caruana et al., 2012 \\
\hline Caryophyllales & Portulacaceae & Portulaca oleracea & $\mathrm{AB}$ & $\mathrm{F}$ & $\begin{array}{l}\text { Direkbysarakom et al., } \\
2004\end{array}$ \\
\hline Commelinales & Pontederiaceae & Eichhornia crassipes & $\mathrm{AB}$ & NA & $\begin{array}{l}\text { Caruso et al., 2013; Chang } \\
\text { et al., } 2013\end{array}$ \\
\hline Cucurbitales & Cucurbitaceae & Cucurbita pepo & NA & W & Caruso et al., 2013 \\
\hline Cucurbitales & Cucurbitaceae & $\begin{array}{l}\text { Mormodica } \\
\text { charantia }\end{array}$ & AV & $\mathrm{R}$ & $\begin{array}{l}\text { Balasubramanian et al., } \\
2007\end{array}$ \\
\hline Cucurbitales & Cucurbitaceae & $\begin{array}{l}\text { Mormodica } \\
\text { cochinchinensis }\end{array}$ & $\mathrm{AP}$ & $\mathrm{L}$ & Wu et al., 2011 \\
\hline Cyatheales & Cyatheaceae & Cyathea kanehirae & $\begin{array}{l}\mathrm{AB} \\
\mathrm{IM}\end{array}$ & $\mathrm{L}$ & Yeh and Chen, 2009 \\
\hline Dipsacales & Caprifoliaceae & Lonicera japonica & $\begin{array}{l}\mathrm{AB} \\
\mathrm{AV} \\
\mathrm{IM}\end{array}$ & $\mathrm{L}$ & $\begin{array}{l}\text { Ardò et al., } 2008 \text {; } \\
\text { Direkbysarakom et al., } \\
2006\end{array}$ \\
\hline Dryopteridales & Dryopteridaceae & $\begin{array}{l}\text { Dryopteris } \\
\text { crassirizhoma }\end{array}$ & AP & NA & Lu et al., 2012 \\
\hline Ericales & Primulaceae & $\begin{array}{l}\text { Aegiceras } \\
\text { corniculatum }\end{array}$ & $\mathrm{AB}$ & NA & Choudhury et al., 2005 \\
\hline Ericales & Theaceae & Camellia sinensis & $\begin{array}{l}\mathrm{AB} \\
\mathrm{AP} \\
\mathrm{GP}\end{array}$ & NA & Sheikhzadeh et al., 2011 \\
\hline Ericales & Lecythidaceae & Cariniana legalis & $\mathrm{AB}$ & NA & Castro et al., 2008 \\
\hline
\end{tabular}


Table 9.1 (Continued)

\begin{tabular}{|c|c|c|c|c|c|}
\hline Order & Family & Species & Activities & $\begin{array}{l}\text { Part } \\
\text { used }\end{array}$ & Reference \\
\hline Ericales & Balsaminaceae & Impatiens biflora & $\begin{array}{l}\mathrm{AB} \\
\mathrm{AV}\end{array}$ & NA & $\begin{array}{l}\text { Direkbysarakom et al., } \\
2004\end{array}$ \\
\hline Ericales & Primulaceae & $\begin{array}{l}\text { Lysimachia } \\
\text { christinae }\end{array}$ & AP & NA & Huang et al., 2013 \\
\hline Ericales & Styracoceae & Styrax japonica & $\mathrm{AB}$ & $\mathrm{S}$ & Harikrishnan et al., 2011g \\
\hline Fabales & Fabaceae & Albizia saman & NA & NA & Caruso et al., 2013 \\
\hline Fabales & Fabaceae & $\begin{array}{l}\text { Astragalus } \\
\text { membranaceus }\end{array}$ & $\begin{array}{l}\mathrm{AB} \\
\mathrm{IM}\end{array}$ & $\mathrm{L}$ & Pan et al., 2013 \\
\hline Fabales & Fabaceae & Caesalpinia sappan & $\mathrm{AP}$ & NA & Huang et al., 2013 \\
\hline Fabales & Fabaceae & Cassia alata & AV & NA & Sivasankar et al., 2015 \\
\hline Fabales & Fabaceae & Cassia fistula & $\mathrm{AV}$ & NA & Sivasankar et al., 2015 \\
\hline Fabales & Fabaceae & Cynometra iripa & $\mathrm{AB}$ & NA & Choudhury et al., 2005 \\
\hline Fabales & Fabaceae & $\begin{array}{l}\text { Falcataria } \\
\text { moluccana }\end{array}$ & NA & NA & Caruso et al., 2013 \\
\hline Fabales & Fabaceae & Gliricidia sepium & NA & NA & Caruso et al., 2014 \\
\hline Fabales & Fabaceae & Glycine $\max$ & NA & $\mathrm{R}$ & Caruso et al., 2015 \\
\hline Fabales & Fabaceae & $\begin{array}{l}\text { Koompassia } \\
\text { malaccensis }\end{array}$ & NA & NA & Caruso et al., 2016 \\
\hline Fabales & Fabaceae & $\begin{array}{l}\text { Leucaena } \\
\text { leucocephala }\end{array}$ & NA & $\mathrm{L}, \mathrm{R}$ & Caruso et al., 2017 \\
\hline Fabales & Fabaceae & Leucaena glauca & AP & W & $\begin{array}{l}\text { Caruso et al., 2013; } \\
\text { Direkbysarakom et al., } \\
2004\end{array}$ \\
\hline Fabales & Fabaceae & Lupinus perennis & NA & NA & Awad and Austin, 2010 \\
\hline Fabales & Fabaceae & Mimosa pudica & AV & NA & Sivasankar et al., 2015 \\
\hline Fabales & Fabaceae & Mucuna pruriens & $\mathrm{AP}$ & W & Ekanem et al., 2004 \\
\hline Fabales & Fabaceae & Parkia speciosa & NA & $\mathrm{L}$ & Caruso et al., 2013 \\
\hline Fabales & Fabaceae & Psoralea corylifolia & $\begin{array}{l}\mathrm{AP} \\
\mathrm{GP}\end{array}$ & $\mathrm{L}$ & Ling et al., 2003 \\
\hline Fabales & Fabaceae & Senna alata & $\begin{array}{l}\mathrm{AB} \\
\mathrm{AV}\end{array}$ & NA & Caruso et al., 2013 \\
\hline Fabales & Fabaceae & Senna siamea & NA & NA & Caruso et al., 2013 \\
\hline Fabales & Fabaceae & $\begin{array}{l}\text { Sesbania } \\
\text { grandiflora }\end{array}$ & NA & NA & Caruso et al., 2013 \\
\hline Fabales & Fabaceae & $\begin{array}{l}\text { Sophora } \\
\text { alopercuroides }\end{array}$ & $\begin{array}{l}\mathrm{AB} \\
\mathrm{AP}\end{array}$ & NA & Yi et al., 2012 \\
\hline Fabales & Fabaceae & Sophora flavescens & $\begin{array}{l}\mathrm{AB} \\
\mathrm{AP}\end{array}$ & NA & $\begin{array}{l}\text { Caruana et al., 2012; Wu } \\
\text { et al., } 2013\end{array}$ \\
\hline Fabales & Fabaceae & Tamarindus indica & NA & $\mathrm{L}, \mathrm{R}$ & Caruso et al., 2013 \\
\hline Fabales & Fabaceae & Tephrosia purpurea & NA & NA & Sivasankar et al., 2015 \\
\hline Fabales & Fabaceae & Trifolium pratense & GP & $\mathrm{S}$ & Syahidah et al., 2015 \\
\hline
\end{tabular}


Table 9.1 (Continued)

\begin{tabular}{|c|c|c|c|c|c|}
\hline Order & Family & Species & Activities & $\begin{array}{l}\text { Part } \\
\text { used }\end{array}$ & Reference \\
\hline Fabales & Polygalaceae & Polygala tenuifolia & AP & NA & Lu et al., 2012 \\
\hline Fabales & Quillajaceae & Quillaja saponaria & GP & NA & Francis et al., 2005 \\
\hline Fagales & Betulaceae & Alnus firma & $\mathrm{AB}$ & NA & Harikrishnan et al., 2011i \\
\hline Gentianales & Apocynaceae & Anathoda vasica & $\begin{array}{l}\mathrm{AB} \\
\mathrm{IM}\end{array}$ & $\mathrm{L}$ & Minomol, 2005 \\
\hline Gentianales & Apocynaceae & Calotropis gigantea & $\begin{array}{l}\mathrm{AB} \\
\mathrm{IM}\end{array}$ & $\mathrm{L}$ & Olusola et al., 2013 \\
\hline Gentianales & Apocynaceae & $\begin{array}{l}\text { Catharanthus } \\
\text { roseus }\end{array}$ & AV & $\mathrm{L}$ & Sivasankar et al., 2015 \\
\hline Gentianales & Apocynaceae & Daemia extensa & $\mathrm{AB}$ & $\mathrm{L}$ & Jinish, 2002 \\
\hline Gentianales & Apocynaceae & Plumeria rubra & NA & NA & Caruso et al., 2013 \\
\hline Gentianales & Rubiaceae & Coffea arabica & NA & NA & Abdel, 2015 \\
\hline Gentianales & Rubiaceae & Morinda citrifolia & NA & NA & Caruso et al., 2013 \\
\hline Lamiales & Acanthaceae & $\begin{array}{l}\text { Andrographis } \\
\text { paniculata }\end{array}$ & $\begin{array}{l}\mathrm{AB} \\
\mathrm{GP}\end{array}$ & NA & $\begin{array}{l}\text { Caruso et al., 2009; } \\
\text { Rattanachaikunsopon and } \\
\text { Phumkhachorn, } 2009\end{array}$ \\
\hline Lamiales & Acanthaceae & $\begin{array}{l}\text { Clinacanthus } \\
\text { nutans }\end{array}$ & $Y$ & W & $\begin{array}{l}\text { Direkbysarakom et al., } \\
1998\end{array}$ \\
\hline Lamiales & Acanthaceae & Hygrophila spinosa & $\begin{array}{l}\mathrm{AB} \\
\mathrm{IM}\end{array}$ & $\mathrm{L}$ & Raja et al., 2012 \\
\hline Lamiales & Lamiaceae & $\begin{array}{l}\text { Leucas } \\
\text { lavandulifolia }\end{array}$ & NA & NA & Caruso et al., 2013 \\
\hline Lamiales & Lamiaceae & Mentha piperita & NA & $\mathrm{W}$ & $\begin{array}{l}\text { Abasali and Mohamad, } \\
2010\end{array}$ \\
\hline Lamiales & Lamiaceae & Ocimum & NA & NA & Sivasankar et al., 2015 \\
\hline Lamiales & Lamiaceae & Ocimum basilicum & GP & $\mathrm{W}$ & Syahidah et al., 2015 \\
\hline Lamiales & Lamiaceae & Ocimum sanctum & $\begin{array}{l}\mathrm{AB} \\
\mathrm{IM} \\
\mathrm{GP}\end{array}$ & NA & $\begin{array}{l}\text { Logambal et al., 2000; } \\
\text { Pajaraj et al., } 2011\end{array}$ \\
\hline Lamiales & Lamiaceae & $\begin{array}{l}\text { Ocimum } \\
\text { tenuiflorum }\end{array}$ & NA & NA & Caruso et al., 2013 \\
\hline Lamiales & Lamiaceae & Origanum monites & AF & W & Syahidah et al., 2015 \\
\hline Lamiales & Lamiaceae & $\begin{array}{l}\text { Origanum } \\
\text { heracleoticum }\end{array}$ & $\mathrm{AB}$ & $\mathrm{W}$ & Zheng et al., 2007 \\
\hline Lamiales & Lamiaceae & $\begin{array}{l}\text { Origanum } \\
\text { minutiflorum }\end{array}$ & AP & $\mathrm{Fr}$ & Karagouni et al., 2005 \\
\hline Lamiales & Lamiaceae & Origanum onites & $\mathrm{AF}$ & $\mathrm{S}$ & Caruso et al., 2013 \\
\hline Lamiales & Lamiaceae & Origanum vulgare & $\begin{array}{l}\mathrm{AB} \\
\mathrm{GP}\end{array}$ & NA & Caruso et al., 2013 \\
\hline Lamiales & Lamiaceae & Prunella vulgaris & $\begin{array}{l}\mathrm{AP} \\
\mathrm{IM}\end{array}$ & $\mathrm{R}$ & Harikrishnan et al., 2011d \\
\hline Lamiales & Lamiaceae & $\begin{array}{l}\text { Rosmarinus } \\
\text { officinalis }\end{array}$ & $\begin{array}{l}\mathrm{AB} \\
\mathrm{AP}\end{array}$ & NA & Abutbul et al., 2004 \\
\hline
\end{tabular}


Table 9.1 (Continued)

\begin{tabular}{|c|c|c|c|c|c|}
\hline Order & Family & Species & Activities & $\begin{array}{l}\text { Part } \\
\text { used }\end{array}$ & Reference \\
\hline Lamiales & Lamiaceae & $\begin{array}{l}\text { Satureja } \\
\text { khuzistanica }\end{array}$ & IM & NA & Khansari et al., 2013 \\
\hline Lamiales & Lamiaceae & $\begin{array}{l}\text { Satureja } \\
\text { bachtiarica }\end{array}$ & $\mathrm{AB}$ & $\mathrm{B}$ & Pirbalouti et al., 2011 \\
\hline Lamiales & Lamiaceae & Satureja thymbra & $\mathrm{AF}$ & B & Syahidah et al., 2015 \\
\hline Lamiales & Lamiaceae & $\begin{array}{l}\text { Scutellaria } \\
\text { baicalensis }\end{array}$ & $\begin{array}{l}\mathrm{AB} \\
\mathrm{IM}\end{array}$ & B & $\begin{array}{l}\text { Harikrishnan et al., 2012b; } \\
\text { Pan et al., } 2013\end{array}$ \\
\hline Lamiales & Lamiaceae & $\begin{array}{l}\text { Plectranthus } \\
\text { scutellarioides }\end{array}$ & NA & W & Caruso et al., 2013 \\
\hline Lamiales & Lamiaceae & Tectona grandis & NA & NA & Caruso et al., 2013 \\
\hline Lamiales & Lamiaceae & Thymbra spicata & $\mathrm{AF}$ & $\mathrm{L}, \mathrm{B}$ & Syahidah et al., 2015 \\
\hline Lamiales & Lamiaceae & Zataria multiflora & $\mathrm{AF}$ & NA & Soltani et al., 2010 \\
\hline Lamiales & Oleaceae & Fructus forsythiae & $\mathrm{AB}$ & $\mathrm{NA}$ & Pan et al., 2013 \\
\hline Lamiales & Oleaceae & $\begin{array}{l}\text { Nyctanthes } \\
\text { arbortristis }\end{array}$ & $\begin{array}{l}\mathrm{AB} \\
\mathrm{IM}\end{array}$ & NA & Kibubakaran et al., 2010 \\
\hline Lamiales & Oleaceae & Olea europea & AV & NA & Micol et al., 2005 \\
\hline Lamiales & Plantaginaceae & Picrorhiza kurrooa & $\mathrm{AB}$ & $\mathrm{L}$ & Raja et al., 2012 \\
\hline Lamiales & Pedaliacae & Sesamum indicum & NA & $\mathrm{L}$ & Dada et al., 2014 \\
\hline Lamiales & Verbenaceae & Lantana camara & $\mathrm{AV}$ & L, S, B & $\begin{array}{l}\text { Balasubramanian et al., } \\
2007\end{array}$ \\
\hline Laurales & Lauraceae & $\begin{array}{l}\text { Cinnamomum } \\
\text { cassia }\end{array}$ & $\begin{array}{l}\mathrm{AB} \\
\mathrm{AP}\end{array}$ & W & Ji et al., 2012 \\
\hline Laurales & Lauraceae & $\begin{array}{l}\text { Cinnamomum } \\
\text { verum }\end{array}$ & $\begin{array}{l}\mathrm{AB} \\
\mathrm{GP}\end{array}$ & $\mathrm{NA}$ & Talpur et al., 2013 \\
\hline Laurales & Lauraceae & $\begin{array}{l}\text { Cinnamomum } \\
\text { zeylanicum }\end{array}$ & $\mathrm{AB}$ & $\mathrm{L}$ & Ahmad et al., 2011 \\
\hline Laurales & Lauraceae & Laurus nobilis & $\begin{array}{l}\mathrm{AB} \\
\mathrm{IM}\end{array}$ & $\mathrm{L}, \mathrm{B}$ & Bilen and Bulut, 2010 \\
\hline Laurales & Lauraceae & Lindera aggregata & $\mathrm{AP}$ & NA & Ji et al., 2012 \\
\hline Laurales & Siparunaceae & Siparuna guianensis & $\mathrm{AB}$ & NA & Castro et al., 2008 \\
\hline Liliales & Melanthiaceae & Paris polyphylla & $\mathrm{NA}$ & $\mathrm{NA}$ & Wang et al., 2010a \\
\hline Magnoliales & Annonaceae & Annona muricata & $\mathrm{AB}$ & $\mathrm{L}$ & Caruso et al., 2013 \\
\hline Magnoliales & Annonaceae & Xylopia aethiopica & NA & NA & Okeke et al., 2001 \\
\hline Magnoliales & Magnoliaceae & Magnolia officinalis & $\begin{array}{l}\mathrm{AV} \\
\mathrm{AF}\end{array}$ & NA & $\begin{array}{l}\text { Caruana et al., 2012; } \\
\text { Huang et al., } 2015\end{array}$ \\
\hline Magnoliales & Myristicaceae & Myristica fragans & $\begin{array}{l}\mathrm{AB} \\
\mathrm{IM}, \\
\mathrm{GP}\end{array}$ & $\mathrm{L}$ & Sivaram et al., 2004 \\
\hline Magnoliales & Myristicaceae & Virola sebifera & $\mathrm{AB}$ & NA & Castro et al., 2008 \\
\hline Malpighiales & Calophyllaceae & $\begin{array}{l}\text { Calophyllum } \\
\text { inophyllum }\end{array}$ & $\mathrm{AV}$ & NA & Sivasankar et al., 2015 \\
\hline
\end{tabular}


Table 9.1 (Continued)

\begin{tabular}{|c|c|c|c|c|c|}
\hline Order & Family & Species & Activities & $\begin{array}{l}\text { Part } \\
\text { used }\end{array}$ & Reference \\
\hline Malpighiales & Euphorbiaceae & Acalypha indica & $\begin{array}{l}\mathrm{AB} \\
\mathrm{AV} \\
\mathrm{IM}\end{array}$ & NA & Raja et al., 2012 \\
\hline Malpighiales & Euphorbiaceae & Acalypha australis & AV & $\mathrm{L}$ & $\begin{array}{l}\text { Direkbysarakom et al., } \\
2004\end{array}$ \\
\hline Malpighiales & Euphorbiaceae & $\begin{array}{l}\text { Actinostemon } \\
\text { concolor }\end{array}$ & $\mathrm{AB}$ & W & Castro et al., 2008 \\
\hline Malpighiales & Euphorbiaceae & Croton floribundus & $\mathrm{AB}$ & NA & Castro et al., 2008 \\
\hline Malpighiales & Euphorbiaceae & Croton zambesicus & AV & NA & Sivasankar et al., 2015 \\
\hline Malpighiales & Euphorbiaceae & $\begin{array}{l}\text { Euphorbia } \\
\text { antiquorum }\end{array}$ & NA & NA & Caruso et al., 2013 \\
\hline Malpighiales & Euphorbiaceae & $\begin{array}{l}\text { Euphorbia } \\
\text { fishceriana }\end{array}$ & $\begin{array}{l}\text { AF, } \\
\text { AP }\end{array}$ & W & Zhang et al., 2014 \\
\hline Malpighiales & Euphorbiaceae & Euphorbia hirta & $\mathrm{AB}$ & NA & $\begin{array}{l}\text { Huang et al., 2015; } \\
\text { Pratheepa and Sukumaran, } \\
2011\end{array}$ \\
\hline Malpighiales & Euphorbiaceae & $\begin{array}{l}\text { Euphorbia } \\
\text { plumerioides }\end{array}$ & NA & NA & Caruso et al., 2013 \\
\hline Malpighiales & Euphorbiaceae & $\begin{array}{l}\text { Euphorbia } \\
\text { thymifolia }\end{array}$ & $\mathrm{AB}$ & NA & $\begin{array}{l}\text { Direkbysarakom et al., } \\
2004\end{array}$ \\
\hline Malpighiales & Euphorbiaceae & Exoecaria agallocha & $\mathrm{AB}$ & W & Dhayanithi et al., 2012 \\
\hline Malpighiales & Euphorbiaceae & Hura crepitans & AV & $\mathrm{L}$ & Sivasankar et al., 2015 \\
\hline Malpighiales & Euphorbiaceae & Manihot esculenta & NA & NA & Caruso et al., 2013 \\
\hline Malpighiales & Euphorbiaceae & Sapium sebiferum & AV & NA & $\begin{array}{l}\text { Direkbysarakom et al., } \\
2004\end{array}$ \\
\hline Malpighiales & Euphorbiaceae & $\begin{array}{l}\text { Tetracarpidium } \\
\text { conophorum }\end{array}$ & $\begin{array}{l}\text { AB, } \\
\text { IM, } \\
\text { GP }\end{array}$ & $\mathrm{Fr}$ & Olusola et al., 2013 \\
\hline Malpighiales & Hypericaceae & $\begin{array}{l}\text { Cratoxylum } \\
\text { formosum }\end{array}$ & $\begin{array}{l}\mathrm{AB} \\
\mathrm{IM} \\
\mathrm{GP}\end{array}$ & NA & $\begin{array}{l}\text { Rattanachaikunsopon and } \\
\text { Phumkhachorn, 2010c }\end{array}$ \\
\hline Malpighiales & Phyllanthaceae & Antidesma bunius & NA & W & Caruso et al., 2013 \\
\hline Malpighiales & Phyllanthaceae & Phyllanthus acidus & NA & $\mathrm{L}, \mathrm{F}$ & Caruso et al., 2013 \\
\hline Malpighiales & Phyllanthaceae & Phyllanthus amarus & $\begin{array}{l}\mathrm{AB} \\
\mathrm{AV}\end{array}$ & $\mathrm{L}$ & $\begin{array}{l}\text { Balasubramanian et al., } \\
2007\end{array}$ \\
\hline Malpighiales & Phyllanthaceae & Phyllanthus niruri & GP & $\mathrm{L}$ & Citarasu et al., 2002 \\
\hline Malpighiales & Phyllanthaceae & Phyllanthus debelis & $\begin{array}{l}\mathrm{AB} \\
\mathrm{AV}\end{array}$ & $\begin{array}{l}\text { L, B, } \\
\text { Fr }\end{array}$ & Sivasankar et al., 2015 \\
\hline Malpighiales & Phyllanthaceae & Phyllanthus emblica & $\begin{array}{l}\text { AV } \\
\text { IM }\end{array}$ & NA & Sivasankar et al., 2015 \\
\hline Malpighiales & Phyllanthaceae & $\begin{array}{l}\text { Phyllanthus } \\
\text { reticulatus }\end{array}$ & $\begin{array}{l}\mathrm{AB} \\
\mathrm{AV}\end{array}$ & W & Sivasankar et al., 2015 \\
\hline Malpighiales & Phyllanthaceae & $\begin{array}{l}\text { Phyllanthus } \\
\text { urinaria }\end{array}$ & $\begin{array}{l}\mathrm{AB} \\
\mathrm{AV}\end{array}$ & R, F, S & Caruso et al., 2013 \\
\hline
\end{tabular}


Table 9.1 (Continued)

\begin{tabular}{|c|c|c|c|c|c|}
\hline Order & Family & Species & Activities & $\begin{array}{l}\text { Part } \\
\text { used }\end{array}$ & Reference \\
\hline Malpighiales & Phyllanthaceae & $\begin{array}{l}\text { Sauropus } \\
\text { androgynus }\end{array}$ & GP & NA & Putra et al., 2013 \\
\hline Malvales & Malvaceae & $\begin{array}{l}\text { Hibiscus } \\
\text { rosa-sinensis }\end{array}$ & $\mathrm{NA}$ & NA & Caruso et al., 2013 \\
\hline microalgae & & Euglena viridis & $\begin{array}{l}\mathrm{AB} \\
\mathrm{IM}\end{array}$ & NA & Das, 2009 \\
\hline Mucorales & Mucoraceae & Massa medicata & $\begin{array}{l}\mathrm{AB} \\
\mathrm{GP}\end{array}$ & NA & Ji et al., 2007a,2007b \\
\hline Myrtales & Combretaceae & Terminalia bellerica & $\mathrm{AB}$ & $\mathrm{NA}$ & Jinish, 2002 \\
\hline Myrtales & Combretaceae & Terminalia cattapa & $\begin{array}{l}\mathrm{AB} \\
\mathrm{AP}\end{array}$ & $\mathrm{S}$ & $\begin{array}{l}\text { Chitmanat et al., 2005; } \\
\text { Purivirojkul, } 2012\end{array}$ \\
\hline Myrtales & Lythraceae & Punica granatum & $\begin{array}{l}\mathrm{AB}, \\
\mathrm{AV} \\
\mathrm{AP} \\
\mathrm{IM}, \\
\mathrm{GP}\end{array}$ & NA & Harikrishnan et al., 2010e \\
\hline Myrtales & Myrtaceae & $\begin{array}{l}\text { Calyptranthes } \\
\text { clusiifolia }\end{array}$ & $\mathrm{AB}$ & W & Castro et al., 2008 \\
\hline Myrtales & Myrtaceae & Myrcia tomentosa & $\mathrm{AB}$ & NA & Castro et al., 2008 \\
\hline Myrtales & Myrtaceae & Psidium guajava & $\begin{array}{l}\mathrm{AB} \\
\mathrm{AV}\end{array}$ & NA & Pachanawan et al., 2008 \\
\hline Myrtales & Myrtaceae & $\begin{array}{l}\text { Syzygium } \\
\text { aromaticum }\end{array}$ & NA & NA & $\begin{array}{l}\text { Rattanachaikunsopon and } \\
\text { Phumkhachorn, 2010; Abd } \\
\text { El-Galil, } 2012\end{array}$ \\
\hline Myrtales & Onagraceae & Epilobium hirsutum & $\mathrm{AB}$ & NA & Pakravan et al., 2011 \\
\hline Myrtales & Onagraceae & Oenothera biennis & $\begin{array}{l}\mathrm{AB} \\
\mathrm{AV}\end{array}$ & $\mathrm{L}, \mathrm{R}$ & $\begin{array}{l}\text { Shangliang et al., } 1990 \\
\text { (sriva) }\end{array}$ \\
\hline Pinales & Pinaceae & Pinus massoniana & $\begin{array}{l}\mathrm{AB} \\
\mathrm{AF} \\
\mathrm{AP}\end{array}$ & $\mathrm{L}, \mathrm{R}$ & $\begin{array}{l}\text { Direkbysarakom et al., } \\
2004\end{array}$ \\
\hline Pinales & Pinaceae & $\begin{array}{l}\text { Pseudolarix } \\
\text { kaempferi }\end{array}$ & $\mathrm{AP}$ & NA & Ji et al., 2012 \\
\hline Piperales & Aristolochiaceae & Aristolochia indica & AV & NA & Sivasankar et al., 2015 \\
\hline Piperales & Piperaceae & Piper betle & $\begin{array}{l}\mathrm{AB} \\
\mathrm{AF}\end{array}$ & NA & Caruso et al., 2013 \\
\hline Piperales & Piperaceae & Piper longum & GP & NA & Citarasu et al., 2002 \\
\hline Piperales & Piperaceae & Piper guineense & $\mathrm{AP}$ & $\mathrm{S}$ & Ekanem et al., 2004 \\
\hline Piperales & Piperaceae & Piper nigrum & IM & $\mathrm{R}$ & Caruso et al., 2013 \\
\hline Poales & Poaceae & Cynodon dactylon & $\begin{array}{l}\mathrm{AB}, \\
\mathrm{AV}, \\
\mathrm{AP} \\
\mathrm{IM}, \\
\mathrm{GP}\end{array}$ & NA & $\begin{array}{l}\text { Balasubramanian et al., } \\
\text { 2007, 2008a,2008b; } \\
\text { Kaleeswaran et al., } 2011\end{array}$ \\
\hline Poales & Poaceae & Oryza sativa & NA & NA & Caruso et al., 2013 \\
\hline
\end{tabular}


Table 9.1 (Continued)

\begin{tabular}{|c|c|c|c|c|c|}
\hline Order & Family & Species & Activities & $\begin{array}{l}\text { Part } \\
\text { used }\end{array}$ & Reference \\
\hline Poales & Poaceae & Panicum repens & NA & $\mathrm{R}$ & Caruso et al., 2014 \\
\hline Poales & Poaceae & Setaria barbata & NA & NA & Caruso et al., 2015 \\
\hline Proteales & Nelumbonaceae & Nelumbo nucifera & NA & NA & $\begin{array}{l}\text { Liu et al., 2004; Shao et al., } \\
2004\end{array}$ \\
\hline Ranuncudales & Menispermaceae & Tinospora cordifolia & $\begin{array}{l}\mathrm{AB} \\
\mathrm{IM}\end{array}$ & Fr & $\begin{array}{l}\text { Alexander et al., 2010; } \\
\text { Sudhakaran et al., } 2006\end{array}$ \\
\hline Ranuncudales & Menispermaceae & Tinospora crispa & NA & W & Sivasankar et al., 2015 \\
\hline Ranuncudales & Menispermaceae & $\begin{array}{l}\text { Tinospora } \\
\text { tuberculata }\end{array}$ & NA & NA & Caruso et al., 2013 \\
\hline Ranuncudales & Papaveraceae & Chelidonium majus & AP & $\mathrm{L}$ & Yao et al., 2011 \\
\hline Ranuncudales & Papaveraceae & $\begin{array}{l}\text { Macleaya } \\
\text { microcarpa }\end{array}$ & $\mathrm{AP}$ & NA & Wang et al., 2010b \\
\hline Ranuncudales & Ranunculaceae & Cimifuga fetida & AP & NA & Wu et al., 2011 \\
\hline Ranuncudales & Ranunculoideae & Nigella sativa & NA & $\begin{array}{l}\mathrm{L}, \mathrm{B}, \\
\mathrm{Fr}\end{array}$ & Dorucu et al., 2009 \\
\hline Ranuncudales & Ranunculaceae & Rhizoma cimigufae & $\mathrm{AP}$ & NA & Wu et al., 2011 \\
\hline Rosales & Moraceae & Artocarpus altilis & NA & W & Caruso et al., 2013 \\
\hline Rosales & Moraceae & $\begin{array}{l}\text { Artocarpus } \\
\text { heterophyllus }\end{array}$ & NA & NA & Caruso et al., 2014 \\
\hline Rosales & Moraceae & Ficus benghalensis & IM & $\mathrm{Fr}$ & Olusola et al., 2013 \\
\hline Rosales & Moraceae & Ficus septica & $\mathrm{AB}$ & NA & Caruso et al., 2013 \\
\hline Rosales & Moraceae & Morus alba & $\begin{array}{l}\mathrm{AV} \\
\mathrm{AP}\end{array}$ & $\mathrm{L}$ & Sivasankar et al., 2015 \\
\hline Rosales & Rosaceae & Crataegi fructus & $\begin{array}{l}\mathrm{AB} \\
\mathrm{GP}\end{array}$ & NA & $\begin{array}{l}\text { Ji et al., 2007b; Takaoka } \\
\text { et al., } 2011\end{array}$ \\
\hline Rosales & Rosaceae & Eriobotrya japonica & $\mathrm{AB}$ & NA & Kim et al., 2011 \\
\hline Rosales & Rosaceae & Prunus amygdalus & $\mathrm{AP}$ & $\mathrm{L}$ & Wu et al., 2011 \\
\hline Rosales & Urticaceae & Urtica dioica & $\begin{array}{l}\mathrm{AB} \\
\mathrm{IM}\end{array}$ & $\mathrm{L}$ & Awad and Austin, 2010 \\
\hline Salviniales & Salviniaceae & Salvinia adnata & NA & NA & Caruso et al., 2013 \\
\hline Santalales & Santalaceae & Santalum album & $\mathrm{AP}$ & NA & Tu et al., 2013 \\
\hline Santalales & Santalaceae & Viscum album & $\begin{array}{l}\mathrm{AB} \\
\mathrm{IM}\end{array}$ & NA & $\begin{array}{l}\text { Park and Choi, 2012; } \\
\text { Sharma et al., } 2010\end{array}$ \\
\hline Sapindales & Anacardiaceae & $\begin{array}{l}\text { Anacardium } \\
\text { occidentale }\end{array}$ & NA & NA & Caruso et al., 2013 \\
\hline Sapindales & Anacardiaceae & Cotinus coggyria & GP & NA & Bilen et al., 2011 \\
\hline Sapindales & Anacardiaceae & Galla chinensis & AV & NA & $\begin{array}{l}\text { Direkbysarakom et al., } \\
2004\end{array}$ \\
\hline Sapindales & Anacardiaceae & Mangifera indica & $\begin{array}{l}\mathrm{AB} \\
\mathrm{IM}\end{array}$ & NA & $\begin{array}{l}\text { Awad and Austin, 2010; } \\
\text { Awad et al., 2011; Sahu } \\
\text { et al., } 2007\end{array}$ \\
\hline Sapindales & Burseraceae & $\begin{array}{l}\text { Commiphora } \\
\text { myrrha }\end{array}$ & $\mathrm{AP}$ & $\mathrm{L}$ & Syahidah et al., 2015 \\
\hline
\end{tabular}


Table 9.1 (Continued)

\begin{tabular}{|c|c|c|c|c|c|}
\hline Order & Family & Species & Activities & $\begin{array}{l}\text { Part } \\
\text { used }\end{array}$ & Reference \\
\hline Sapindales & Meliaceae & Azadirachta indica & $\mathrm{AB}$ & NA & Talpur et al., 2013 \\
\hline Sapindales & Meliaceae & $\begin{array}{l}\text { Dysoxylum } \\
\text { gaudichaudianum }\end{array}$ & NA & NA & Caruso et al., 2013 \\
\hline Sapindales & Meliaceae & Melia azedarach & $\begin{array}{l}\mathrm{AV} \\
\mathrm{AP}\end{array}$ & NA & $\begin{array}{l}\text { Caruso et al., 2013; } \\
\text { Sivasankar et al., } 2015\end{array}$ \\
\hline Sapindales & Meliaceae & Toona sinensis & $\begin{array}{l}\mathrm{AB} \\
\mathrm{IM}\end{array}$ & $\mathrm{L}$ & Wu et al., 2010 \\
\hline Sapindales & Rutaceae & Aegle marmelos & $\begin{array}{l}\mathrm{AB} \\
\mathrm{AV} \\
\mathrm{AP}\end{array}$ & NA & $\begin{array}{l}\text { Balasubramanian et al., } \\
\text { 2007; Pratheepa et al., } \\
2010\end{array}$ \\
\hline Sapindales & Rutaceae & $\begin{array}{l}\text { Murraya } \\
\text { paniculata }\end{array}$ & NA & W & Caruso et al., 2013 \\
\hline Sapindales & Rutaceae & Ruta graveolens & AF & $\mathrm{R}$ & $\begin{array}{l}\text { Caruso et al., 2013; } \\
\text { Hashemi-Karouei et al., } \\
2011\end{array}$ \\
\hline Sapindales & Rutaceae & $\begin{array}{l}\text { Zanthoxylum } \\
\text { schinifolium }\end{array}$ & $\begin{array}{l}\text { AP, } \\
\text { IM, }\end{array}$ & $\mathrm{L}$ & Harikrishnan et al., 2010d \\
\hline Sapindales & Simaroubaceae & Brucea javanica & NA & W & Wang et al., 2011 \\
\hline Solanales & Convolvulaceae & Cuscuta chinensis & $\mathrm{AP}$ & $\mathrm{R}$ & Huang et al., 2013 \\
\hline Solanales & Convolvulaceae & Ipomoea aquatica & NA & $\mathrm{R}$ & Caruso et al., 2013 \\
\hline Solanales & Convolvulaceae & Ipomomea batatas & NA & $\mathrm{R}$ & Caruso et al., 2013 \\
\hline Solanales & Convolvulaceae & $\begin{array}{l}\text { Merremia } \\
\text { tomentosa }\end{array}$ & $\mathrm{AB}$ & $\mathrm{R}$ & Castro et al., 2008 \\
\hline Solanales & Solanaceae & $\begin{array}{l}\text { Brugmansia } \\
\text { suaveolens }\end{array}$ & $\mathrm{AB}$ & NA & Caruso et al., 2013 \\
\hline Solanales & Solanaceae & Capsicum frutescens & AP & NA & Ling et al., 2012 \\
\hline Solanales & Solanaceae & Physalis angulata & NA & NA & Caruso et al., 2013 \\
\hline Solanales & Solanaceae & Solanum nigurm & NA & NA & Rajendiran et al., 2008 \\
\hline Solanales & Solanaceae & Solanum suratense & $\mathrm{AB}$ & $\mathrm{R}$ & Jinish, 2002 \\
\hline Solanales & Solanaceae & Solanum trilobatum & $\begin{array}{l}\mathrm{AB} \\
\mathrm{IM} \\
\mathrm{GP}\end{array}$ & NA & $\begin{array}{l}\text { Divyagnaneswari et al., } \\
2007\end{array}$ \\
\hline Solanales & Solanaceae & Withania somnifera & $\begin{array}{l}\mathrm{AB}, \\
\mathrm{AV} \\
\mathrm{IM}, \\
\mathrm{GP}\end{array}$ & NA & Sharma et al., 2010 \\
\hline Vitales & Vitaceae & Cayrathia japonicus & AV & NA & $\begin{array}{l}\text { Direkbysarakom et al., } \\
2004\end{array}$ \\
\hline Vitales & Vitaceae & $\begin{array}{l}\text { Cissus } \\
\text { quadrangularis }\end{array}$ & GP & NA & Radhakrishnan et al., 2014 \\
\hline Zingiberales & Zingiberaceae & Curcuma longa & $\begin{array}{l}\mathrm{AB} \\
\mathrm{AF} \\
\mathrm{IM}\end{array}$ & NA & $\begin{array}{l}\text { Caruso et al., 2013; Sahu } \\
\text { et al., } 2008\end{array}$ \\
\hline
\end{tabular}


Table 9.1 (Continued)

\begin{tabular}{|c|c|c|c|c|c|}
\hline Order & Family & Species & Activities & $\begin{array}{l}\text { Part } \\
\text { used }\end{array}$ & Reference \\
\hline Zingiberales & Zingiberaceae & Curcuma mangga & $\mathrm{AB}$ & NA & Caruso et al., 2013 \\
\hline Zingiberales & Zingiberaceae & $\begin{array}{l}\text { Curcuma } \\
\text { zanthorrhiza }\end{array}$ & NA & NA & Caruso et al., 2014 \\
\hline Zingiberales & Zingiberaceae & Curcuma zeodaria & $\mathrm{AB}, \mathrm{AF}$ & NA & Caruso et al., 2015 \\
\hline Zingiberales & Zingiberaceae & $\begin{array}{l}\text { Etlingera } \\
\text { hemisphaerica }\end{array}$ & NA & NA & Caruso et al., 2016 \\
\hline Zingiberales & Musaceae & Musa acuminata & NA & NA & Caruso et al., 2017 \\
\hline Zingiberales & Musaceae & Musa balbisiana & NA & NA & Caruso et al., 2018 \\
\hline Zingiberales & Musaceae & Musa paradisiace & NA & NA & Caruso et al., 2019 \\
\hline Zingiberales & Zingiberaceae & Zingiber officinale & $\begin{array}{l}\mathrm{AB}, \mathrm{AV} \\
\mathrm{AF}, \mathrm{AP} \\
\mathrm{IM}, \mathrm{GP}\end{array}$ & NA & $\begin{array}{l}\text { Dügenci et al., 2003; } \\
\text { Haghighi and Rohani, } \\
\text { 2013; Nya and Austin, } \\
2009\end{array}$ \\
\hline Algae & red & $\begin{array}{l}\text { Asparagopsis } \\
\text { taxiformis }\end{array}$ & $\begin{array}{l}\mathrm{AB}, \mathrm{AF} \\
\mathrm{AP}\end{array}$ & NA & $\begin{array}{l}\text { Genovese et al., 2012; } \\
\text { Hutson et al., 2012; } \\
\text { Manilal et al., } 2012\end{array}$ \\
\hline Algae & brown & $\begin{array}{l}\text { Himanthalia } \\
\text { elongata }\end{array}$ & $\mathrm{AB}$ & NA & Cox et al., 2010 \\
\hline Algae & brown & Laminaria digitata & $\mathrm{AB}$ & NA & Dubber and Harder, 2008 \\
\hline Algae & red & Ceramium rubrum & $\mathrm{AB}$ & NA & Dubber and Harder, 2009 \\
\hline Algae & red & Gracilaria edulis & $\mathrm{AB}$ & NA & Kolanjinathan et al., 2009 \\
\hline Algae & red & Gracilaria fisheri & NA & NA & Harikrishnan et al., 2011a \\
\hline Algae & red & $\begin{array}{l}\text { Gracilaria } \\
\text { tenuistipitata }\end{array}$ & $\mathrm{AB}, \mathrm{AV}$ & NA & Sirirustananun et al., 2011 \\
\hline Algae & red & Gelidium amansii & $\mathrm{AB}, \mathrm{IM}$ & NA & Fu et al., 2007 \\
\hline Algae & brown & $\begin{array}{l}\text { Sargassum } \\
\text { fusiforme }\end{array}$ & $\mathrm{AB}, \mathrm{IM}$ & NA & Huang et al., 2006 \\
\hline Algae & brown & $\begin{array}{l}\text { Sargassum } \\
\text { duplicatum }\end{array}$ & $\mathrm{AB}, \mathrm{IM}$ & NA & Yeh et al., 2006 \\
\hline Algae & red & $\begin{array}{l}\text { Porphyridium } \\
\text { cruentum }\end{array}$ & $\mathrm{AB}$ & NA & Diaz-Rosales et al., 2008 \\
\hline Algae & green & Ulva sp. & $\mathrm{AP}$ & NA & Hutson et al., 2012 \\
\hline Mushroom & & Citrus paradisi & $\mathrm{AB}$ & NA & Oladosu-Ayayi et al., 2013 \\
\hline Mushroom & & $\begin{array}{l}\text { Ganoderma } \\
\text { lucidum }\end{array}$ & $\mathrm{AB}, \mathrm{IM}$ & NA & Olusola et al., 2013 \\
\hline Mushroom & & $\begin{array}{l}\text { Hericium } \\
\text { erinaceum }\end{array}$ & AP, IM & NA & Harikrishnan et al., 2011b \\
\hline Mushroom & & Inonotus obliquus & $\mathrm{AB}$ & NA & Harikrishnan et al., 2012a \\
\hline Mushroom & & Volvariella volvacea & & NA & Caruso et al., 2013 \\
\hline
\end{tabular}

$\mathrm{AV}$, antiviral; $\mathrm{AB}$, antibacterial; AF, antifungal; AP, antiparasitic; F, flowers; Fr, fruit; GP, growth promoter; IM, immunostimulant; L, leaves; NA, not available; R, root; S, seeds; W, whole plant. 
Most of the plants used both by fish farmers and in scientific studies for potential use in aquaculture correspond to plants widely used in traditional medicine. We identified over 250 plant species from 75 families and 32 orders, which have been used or reported as potentially interesting in aquaculture.

\section{Plant Orders Most Frequently Used in Aquaculture}

Plants from the order Lamiales (family Lamiaceae) were the most studied (12\%), followed by the Fabales (family Fabaceae, 11\%), Asterales (family Asteraceae, 10\%) and Malpighiales (family Euphorbiaceae et Phyllanthaceae, 10\%) (Figure 9.2).

\section{Lamiales}

The Lamiales order comprises about 20 families of dicotyledonous flowering plants (Allaby, 2012). Lamiaceae plants are frequently aromatic and include many culinary herbs such as basil (Ocimum sp.), mint (Mentha sp.), oregano (Origanum sp.), rosemary (Rosmarinus officinalis) and savory (Satureja sp.). Lamiaceae plants are widely used in traditional medicine for treatment of diseases such as colds, headaches, stomach disorders, inflammation and heart disease (Pattanayak et al., 2010). Several essential oils have been reported to display antimicrobial, antispasmodic, carminative and antiviral activity (Mimica-Dukic and Bozin, 2008).

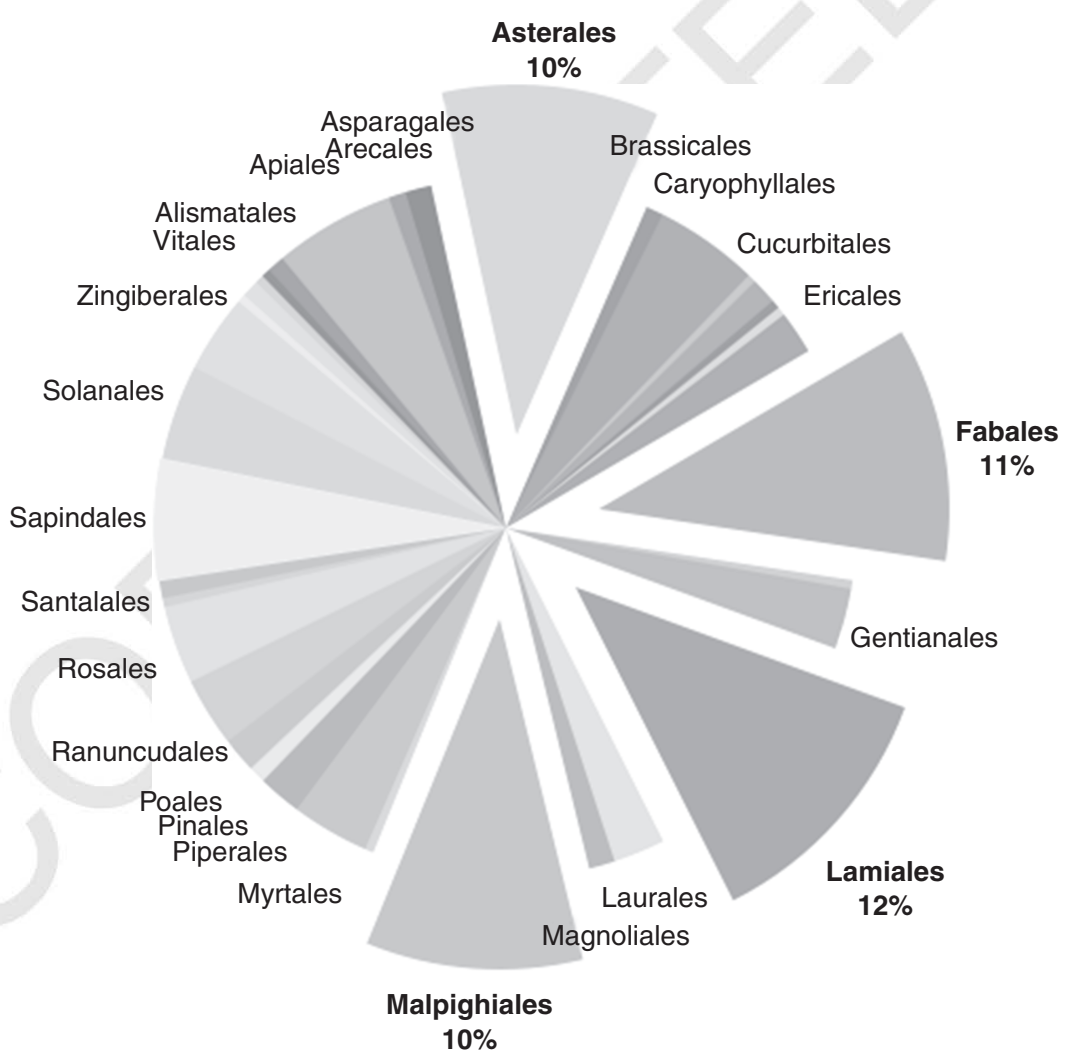

Figure 9.2 Plant orders used in aquaculture.

Orders of plants 


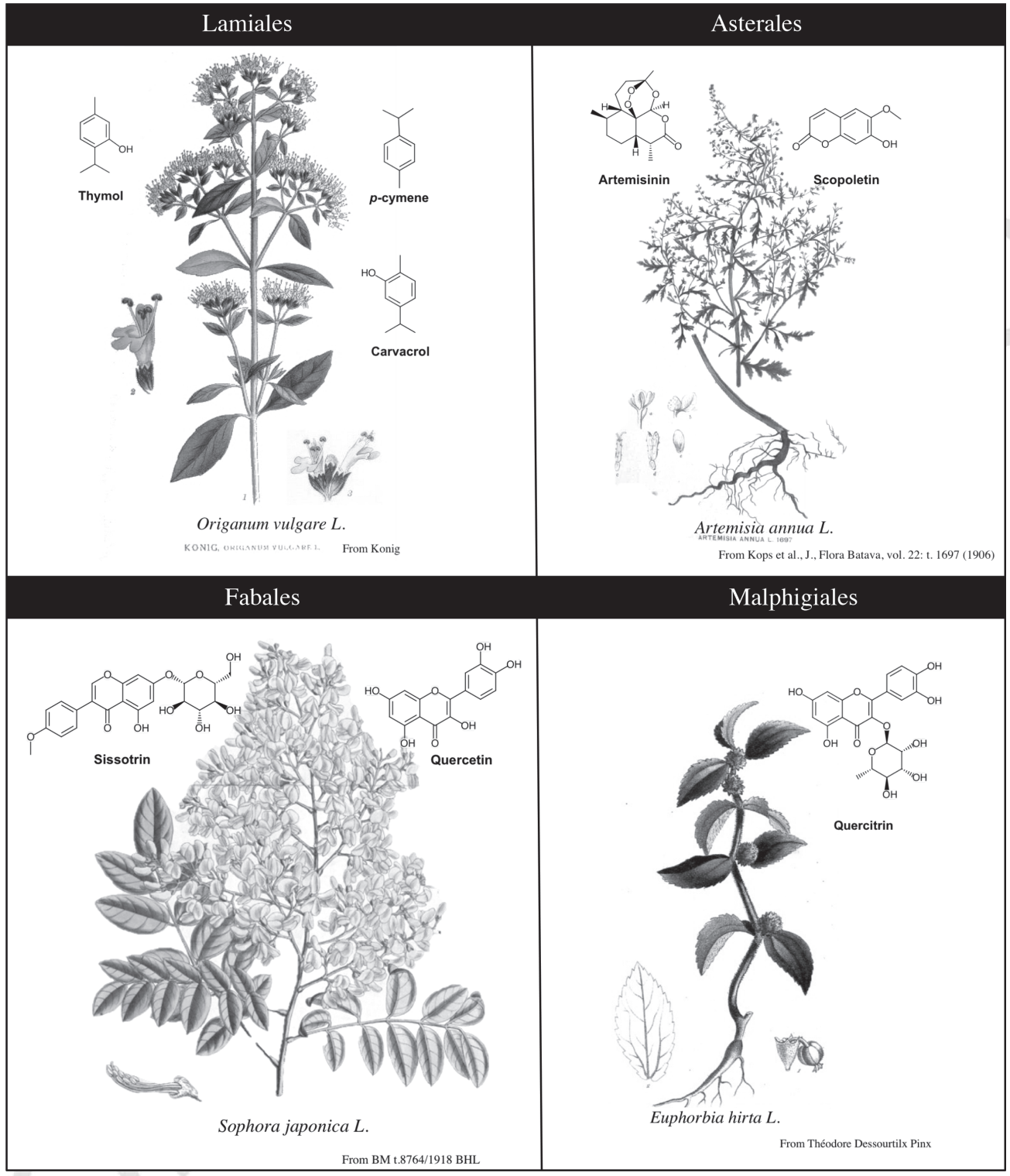

Figure 9.3 Plant examples and isolated molecules from the plant orders most used in aquaculture.

Lamiaceae plants contain a wide range of bioactive metabolites which include numerous monoterpenes and sesquiterpenes such as linalool, geraniol, eugenol, ocimene, carvacrol, p-cymene, thymol and myrcene and flavonoids like luteolin first isolated from Salvia tomentosa (Bower et al., 2014; Malmir et al., 2015; Mimica-Dukic and Bozin, 2008) (Figure 9.3). Plants from the family Lamiaceae also contain several bioactive fatty acids such as palmitic acid and myristic acid and phenolic actives such as rosmarinic acid (Pattanayak et al., 2010). Rattanachaikunsopon and Phumkhachorn (2010) and Abd-El-Galil and Hashiem (2012) showed that antibacterial activity of carvacrol against Edwarsiella tarda and Tenacibaculum 
maritimum was enhanced when administered with cymene (see Figure 9.3). Gormez and Diler (2014) showed that essential oils of Origanum onites and Thymbra spicata were more bioactive against the fungus Saprolegnia parasitica than thymol or carvacrol alone. The latter studies indicate that synergistic effects between different bioactive compounds may exist and enhance plant bioactivity. Hao et al. (2012) found that palmitic acid was effective against the monogenean parasite $D$. intermedium in goldfish (Carassius auratus).

\section{Fabales}

The Fabales include the family Fabaceae, commonly known as the pea or bean family. The Fabaceae is the third largest plant family as it includes more than 12000 plants (Allaby, 2012). Several plants from the family Fabaceae are used in traditional medicine for treatments against diarrhoea, parasite infections, inflammations, rheumatism and ulcers (Molares and Ladio, 2011). Several plants from the Fabaceae family (Albizia saman, Falcataria moluccana, Gliricidia sepum, Koompassia malaccensis, Leucaena leucocephala, L. glauca, Parkia speciosa, Senna alata, S. siamea, Sesbania grandiflora and Tamarindus indica) are used by Indonesian fish farmers to improve fish fitness and treat fish infections (Caruso et al., 2013). Plants from the genus Sophora contain many phytoconstituents with pharmacological and therapeutic properties, including antioxidant, anticancer, antimicrobial, antiviral and anti-inflammatory (Abdelhady et al., 2015). Several polyphenolic compounds have been identified in Sophora japonica, such as tamarixetin, sissotrin, rutin, gallic acid and quercetin (Abdelhady et al., 2015) (see Figure 9.3). Liu et al. (2011) characterized the alkaloids matrine, oxymatrine, sophoridine, oxysophocarpine and sophocarpine from Sophora flavescens. Wu et al. (2013) showed that a diet supplemented with Sophora flavescens enhanced the non-specific immune system of Nile tilapia (Oreochromis niloticus) and increased disease resistance against Streptococcus agalactiae.

Asterales

Asterales is an order of dicotyledonous flowering plants that includes the large family Asteraceae, commonly known as the daisy family. The Asteraceae family contains more than 23000 species; most members are herbaceous but a significant number are shrubs, vines or trees (Allaby, 2012).

Many Artemisia species are present in the Chinese pharmacopoeia, and they have been used as scholeretic, anti-inflammatory and diuretic agents in the treatment of diseases like epidemic hepatitis and fevers (Tang et al., 1992). The use of Artemisia annua in Chinese traditional medicine was recorded before $168 \mathrm{BC}$, and in 1971 artemisinin, a sesquiterpene lactone with antimalarial properties, was isolated (Cávar et al., 2012; Klayman, 1985). Artemisinin and its semi-synthetic derivatives are currently used worldwide for treatment against the malaria parasite Plasmodium falciparum (WHO, 2006) (see Figure 9.3). Artemisia plants also contain abundant monoterpenes (eucalyptol, germacrenes, camphor), coumarins (scopoletin), chlorogenic acids (quinic acid, caffeic acid) and flavonoids (luteolin, isovitexin) (Carbonara et al., 2012; Cávar et al., 2012; Efferth et al., 2011; Juteau et al., 2002) (see Figure 9.3). Artemisia sp. antibacterial and antiparasitic effects against aquaculture pathogens have also been studied. Ekanem and Brisibe (2010) showed the efficacy of ethanol extract of Artemisia annua against the monogenean parasite Heterobranchus longifilis. Huang et al. (2013) reported on the anthelminthic activity of Artemisia argyi against D. intermedius in goldfish. El-Deen and Mohamed (2009) demonstrated the efficacy of Artemisia vulgaris crude extracts against the ectoparasite Trichodina sp. and the bacterium Aeromonas hydrophila in Nile tilapia. 
Aucklandia lappa is another plant from the Asteraceae family with numerous bioactivities and is widely used in traditional medicine for the treatment of asthma, rheumatism, coughs, tuberculosis and many other diseases. (Seo and Shin, 2015; Zhang et al., 2014). A. lappa contains the sesquiterpene lactones costunolide, dehydrocostus lactone and alantolactone which are known to display multiple bioactivities (Li et al., 2005; Seo and Shin, 2015). Xue-Gang et al. (2013) showed that A. lappa displayed antifungal activity against Saprolegnia sp. and Achlya klebsiana.

Other Asteraceae plants have been shown to display interesting bioactivities relevant to application in aquaculture. Oral administration of Eclipta alba in tilapia (Oreochromis mossambicus) enhanced non-specific immune parameters and reduced fish mortality when challenged with $A$. hydrophila (Christybapita et al., 2007). A Siegesbeckia glabrescens-enriched diet displayed an immunomodulatory effect and increased disease resistance in kelp grouper (Epinephelus bruneus) (Harikrishnan et al., 2012b). Artemisia cina, Matricaria chamomilla and Tridax procumbens promoted weight gain and enchanced non-specific immune responses in fish (Abdelhadi et al., 2010; Sivasankar et al., 2015). Echinacea purpura appeared to have immunostimulant, antibacterial and antifungal properties in fish (Aly and Mohamed, 2010; Caruana et al., 2012).

Malpighiales

Malpighiales is a very diverse order, containing the flowering plant families Euphorbiaceae and Phyllanthaceae. Euphorbiaceae, the spurge family, is constituted mainly of herbs but also includes some shrubs and trees. The spurge family contains numerous species of medicinal plants such as different species from the genus Euphorbia or the irritant mangrove Exoecaria agallocha (Ernst et al., 2015). The study of the natural products of the Euphorbia species started in 1968 with the isolation of a tumour-promoting phorbol-12,13-diester from Croton tiglium L., and description of the antitumour activity of a diterpene isolated from Euphorbia esula L. in 1976 (Hecker, 1968; Kupchan, 1976). More recently, a diterpene drug (ingenol mebutate, Picato $\left.{ }^{\circledR}\right)$ isolated from Euphorbia peplus was released for the treatment of actinic keratosis (Berman, 2012).

Euphorbia sp. contains several terpenes, such as the ingol-type diterpenes, euphorantins, flavonoids like quercitrin and tannins (Qi et al., 2014, Trinh and Le, 2014) (see Figure 9.3). Different species of Euphorbia have displayed antibacterial, antiviral, antifungal and antiparastic activities against fish and shellfish pathogens (Direkbusarakom, 2004; Huang et al., 2015; Zhang et al., 2014).

The Phyllanthaceae family is a small (about 2000 species) but diverse family, which includes the genus Phyllanthus. Phyllanthus plants are well known for their potent medicinal activity and are used worldwide (Kumar et al., 2015). Numerous bioactive phytochemicals have been isolated from Phyllanthus plants, including phyllanthin, phyltetralin, phyllangin, corilangin, gallic acid, methylgallate, and rhamnocitrin, protocatechuic acid, kaempferol 3-O-rutinoside, quercitrin and rutin (Fang et al., 2008; Kumar et al., 2015; Wei et al., 2004). Phyllanthus possess a high antibacterial and antiviral potential against pathogens in aquaculture (Balasubramanian et al., 2007; Punitha et al., 2008; Sivasankar et al., 2015).

\section{Plant Species Most Widely Used in Aquaculture}

The plant species that have displayed the highest potential for use in aquaculture are garlic (Allium sativum), pomegranate (Punica granatum), bermuda grass (Cynodon dactylon), Indian ginseng (Whitania somnifera) and ginger (Zingiber officinale). 
Garlic (Asparagales, Amaryllidaceae) has been used by humans for over 7000 years for both culinary and medicinal purposes. Antibacterial, antiparasitic, antioxidant, immunostimulant and growth-promoting activities have been observed in fish and shellfish (Lee and Gao, 2012). Some studies have studied the effect of the pure garlic components allicin and ajoene in aquaculture and demonstrated their immunostimulant capacity and effectiveness against pathogenic fish protozoa Spironucleus vortens, Ichthyophthirius multifiliis and the bacteria $A$. hydrophila (Millet et al., 2011; Nya et al., 2010; Tanekhy and Fall, 2015).

Pomegranate (Myrtales, Lythraceae) has also been used for medical purposes since ancient times. Pomegranates contain numerous phytochemicals such as the bioactive polyphenol ellagitannins which exert antioxidant and anti-inflammatory effects. In aquaculture, studies have shown antibacterial, antiviral, antiparasitic, immunostimulant and growth-promoting activities (Harikrishnan et al., 2010a,2010b; Pirbalouti et al., 2011).

The chemical composition of Bermuda grass (Poales, Poaceae) includes phenolic compounds (gallic acid), tannins (cathechins), anthocyanins (cyanidin) and flavonoids (quercetin) (Khlifi et al., 2013). C. dactylon displays antibacterial, antiviral, antiparasitic, immunostimulant and growth-promoting activities in fish and shellfish (Balasubramanian et al., 2008a,2008b; Kaleeswaran et al., 2011).

Indian ginseng (Solanales, Solanaceae) has been used for centuries in Ayurvedic medicine to increase longevity and vitality. Scientific research has shown antioxidant, anti-inflammatory, immune-modulating and antistress properties in the whole plant extract. W. somnifera biologically active chemical constituents include alkaloids (isopelletierine, anaferine), steroidal lactones (withanolides, withaferins) and saponins containing an additional acyl group (sitoindoside VII and VIII) (Mishra et al., 2000). W. somnifera has antibacterial, antiviral, immunostimulant and growth-promoting activities in aquaculture (Sharma et al., 2010; Talpur et al., 2013; Yogeeswaran et al., 2012).

Ginger (Zingiberales, Zingiberaceae) is a herbaceous perennial plant used for culinary and medicinal purposes. Ginger contains a mixture of zingerone, shogaols and gingerols as well as some sesquiterpenoids, with (-)-zingiberene as the main component (Ali et al., 2008). Bioactivities include antioxidant, anti-inflammatory, antibacterial and apoptosis induction (Dugasani et al., 2010; El-Ghorab et al., 2010). In aquaculture, enrichment of diets with ginger has been shown to promote growth and immunostimulation as well as antibacterial, antiviral, antifungal and antiparasitic activities (Caruana et al., 2012; Kanani et al., 2014; Nya and Austin, 2009; Rajeswari et al., 2012).

\section{Analysis of Plant Bioactivity}

Analysis of plant bioactivity showed that $36 \%$ of the plants studied presented antibacterial activity, whereas $17 \%$ had antiparasitic activity, 16\% immunostimulant activity, $14 \%$ antiviral activity, $13 \%$ growth promoter and only $4 \%$ showed antifungal activity (Figure 9.4). Nevertheless, it should be taken into account that those proportions are biased by the number of studies performed to date on each targeted pathology; for example many more studies have been focused on antibacterial activities than antifungal activities.

Plant bioactivies were also analysed by plant order and we found that Asterales was the order with the highest number of bioactive plants (34), with nearly half of them displaying antibacterial activity (Figure 9.5). Lamiales and Malpighiales were the next two highest (33 and 32). The Lamiales order had the highest number of plants displaying immunostimulant activity (7) and antifungal activity (5), while the Malpighiales presented the highest antiviral activities (11) (see Figure 9.5). 


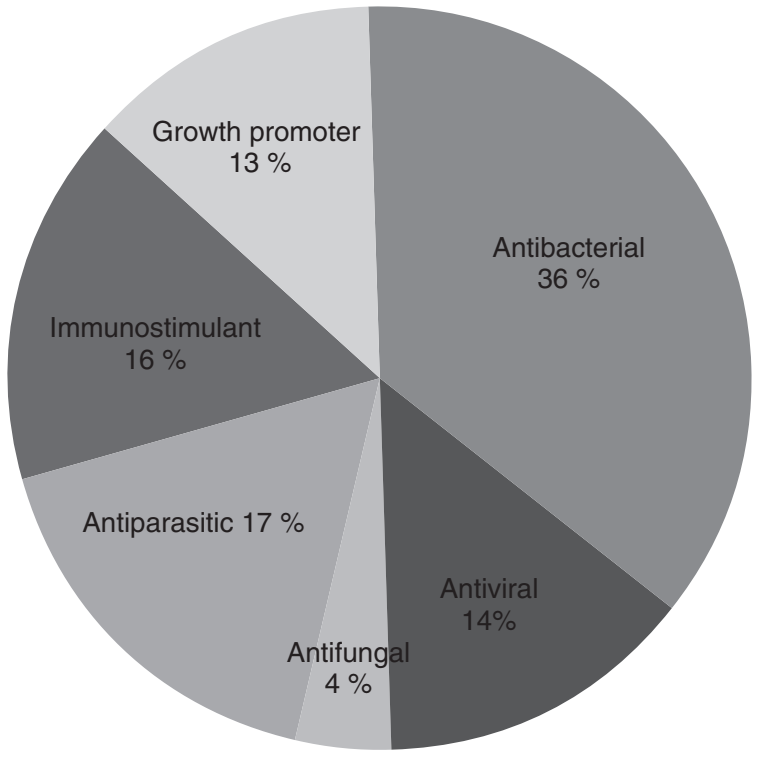

Figure 9.4 Plant bioactivities in aquaculture.

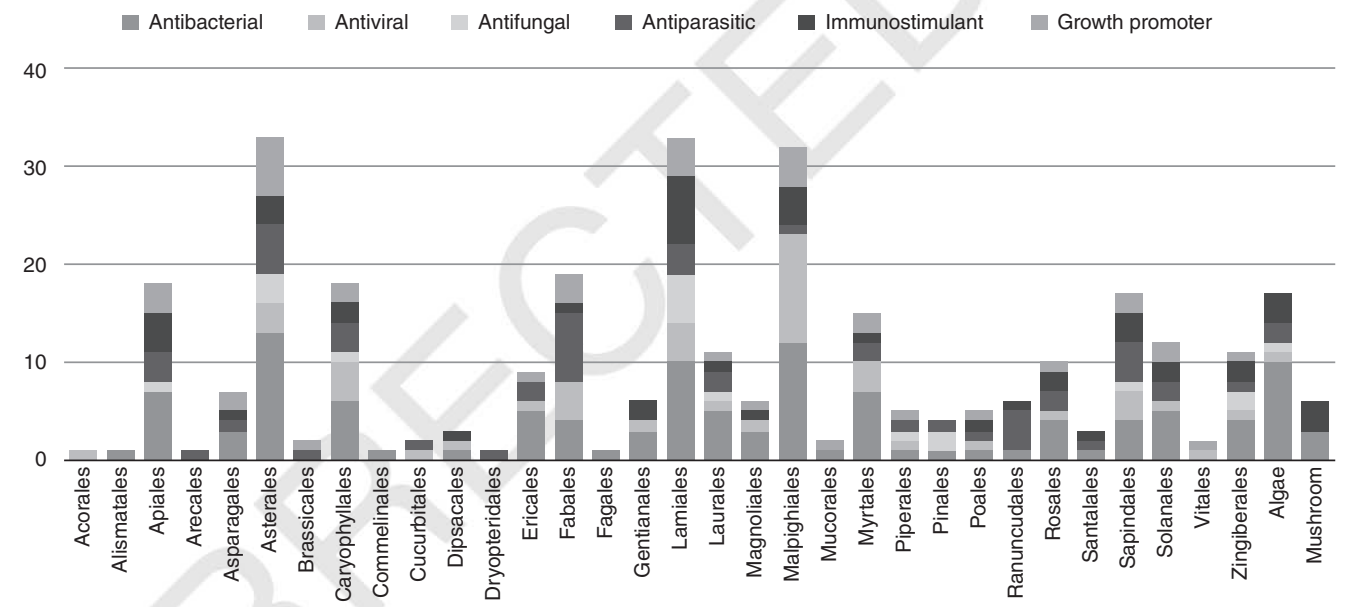

Figure 9.5 Plant order bioactivities in aquaculture.

Bioactivities of plant orders in aquaculture.

Analysis of Plant Parts Used in Aquaculture

Most studies on the use of medicinal plants in aquaculture used plant leaves (37\%), while 22\% used the whole plant as powder, plant essential oil or extract. Root was also often used, at 18\%, followed by seeds (8\%), barks (6\%), fruits (6\%) and finally flowers (4\%) (Figure 9.6).

\section{Other Plants and Perspectives}

Some algae and some mushrooms have also been studied for their potential application in aquaculture. Algae are considered to be a rich source of bioactive molecules; most of the studied algae presented high antibacterial activities, and some also presented immunostimulant, antifungal, antiparasitic and antiviral activity (Choudhury et al., 2005; Genovese 
Figure 9.6 Parts of plants used in aquaculture.

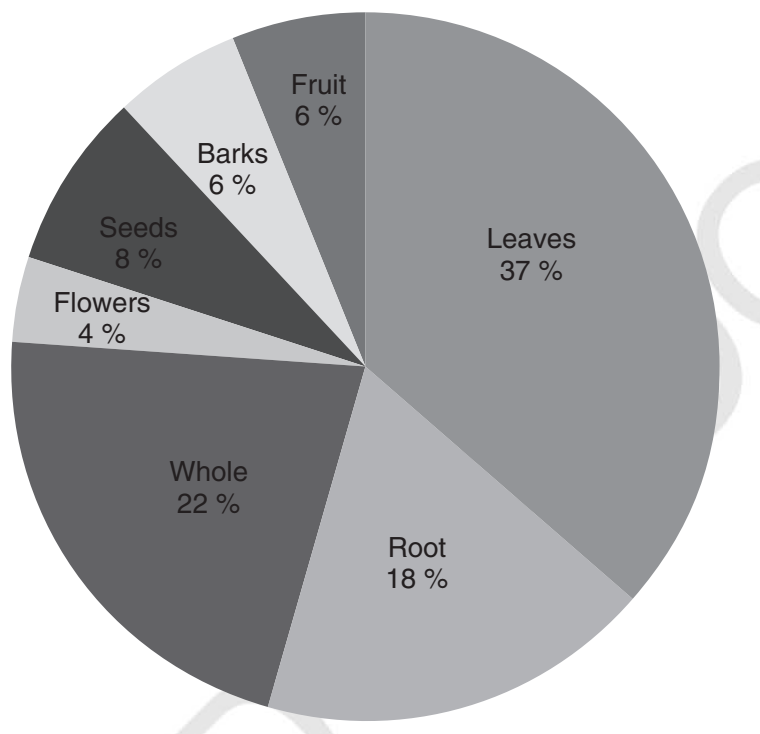

et al., 2013 ; Hutson et al., 2012; Sukoso et al., 2012; Zbakh et al., 2012). For example, the red alga Asparagopsis taxiformis, which is known to produce a high diversity of halogenated metabolites, displayed antibacterial, antifungal and antiparasitic activities against several fish pathogens (Genovese et al., 2012, 2013). Also, A. taxiformis enhanced the immune system of Penaeus monodon and was highly efficient in the treatment of vibriosis in P. monodon (Manilal et al., 2012, 2013). Also, studies like that of Mai et al, (2015) are starting to show interesting properties of other marine organisms such as sponges, which can inhibit quorum sensing of marine pathogenic bacteria such as Vibrio harveyi. Nevertheless, more research needs to be performed in this field, in order to establish the possibilities of application in aquaculture disease management.

Finally, there are numerous plants used in human medicine that have not yet been tested on aquaculture. For example, the gum rockrose (Cistus ladanifer, Cistaceae, Malvales) is a plant widely used in several Mediterranean countries which possesses anti-inflammatory, antibacterial and antioxidant activity (Ferreira et al., 2012; Neves et al., 2009). Dandelion (Taraxacum officinale, Asteraceae, Asterales) has long been used in the northern hemisphere for its diuretic, choleretic, anti-inflammatory, antioxidative, anticarcinogenic, analgesic, antihyperglycaemic, anticoagulatory and prebiotic effects (Schütz et al., 2006).

\section{Conclusion}

Medicinal plants (including algae and mushrooms) present promising potential for use in aquaculture as a substitute for chemotherapy in the treatment of disease outbreaks. Ethnobotanical studies have been highly useful in the discovery of bioactive plants and natural products with interesting applications in aquaculture. However, there is still little knowledge on the mode of action of most bioactive plants, as well as the most suitable form for effective and safe administration. More research is needed to elucidate plant products and their modes of action (to establish the bioactive parts of the plant and the most suitable preparations), and to 
test plant effects on the organism's physiology in order to establish an appropriate treatment strategy (route of administration, dose and length). In addition, research in this field could also benefit greatly from the traditional knowledge of fish farmers who regularly use plants.

Finally, most studies have focused on the potential of terrestrial plants in aquaculture. Although these plants do offer interesting biological properties, they are not a natural component of either fish alimentation or the marine environment. Thus, continuous introduction of plant extracts and their bioactive compounds (through bath treatment or food diffusion) into the marine or freshwater environment could eventually cause environmental problems. Therefore, the use of bioactive algae and seaweeds from local natural environments where aquaculture is carried out could be a better alternative to avoid introduction of exogenous molecules into the environment. Algae are considered to be a rich source of original bioactive molecules which display multiple bioactivities. In aquaculture, several recent studies have showed the potential of algae for the treatment of pathogens or to improve fish fitness. However, they can also cause undesirable effects and toxicity in fish, so more research needs to be performed on the physiological effects of algae on fish and suitable dosing levels (Manilal et al., 2010).

\section{References}

Abasali, H. and Mohamad, S. (2010) Immune response of common carp (Cyprinus carpio) fed with herbal immunostimulants diets. Agricultural Journal, 5, 163-172.

Abd El-Galil, M.A.A. and Hashiem, M. (2012) Experimental infection of tenacibaculosis and a trial for treatment by plant extract carvacrol in surge wrasses fish (Thalassoma Purpureum). Life Science Journal, 9, 442-447.

Abdel-Tawwab, M. (2012) The use of American ginseng (Panax quinquefolium) in practical diets for Nile tilapia (Oreochromis niloticus): growth performance and challenge with Aeromonas hydrophila. Journal of Applied Aquaculture, 24, 366-376.

Abdel-Tawwab, M., Sharafeldin, K.M., Mosaad, M.N.M. and Ismaiel, N.E.M. (2015) Coffee bean in common carp, Cyprinus carpio L. diets: effect on growth performance, biochemical status, and resistance to waterborne zinc toxicity. Aquaculture, 448, 207-213.

Abdelhadi, Y., Saleh, O. and Sakr, S. (2010) Study on the effect of wormseed plants; Artemisia cina L. and chamomile; Matricaria chamomilla L. on growth parameters and immune response of African catfish, Clarias gariepinus. Journal of Fisheries International, 5, 1-7.

Abdelhady, M.I.S., Kamal, A.M., Othman, S.M., Mubarak, M.S. and Hadda, T.B. (2015) Total polyphenolic content, antioxidant, cytotoxic, antidiabetic activities, and polyphenolic compounds of Sophora japonica grown in Egypt. Medicinal Chemistry Research, 24, 482-495.

Abutbul, S., Golan-Goldhirsh, A., Baranzani, O. and Zilberg, D. (2004) Use of Rosmarinus officinalis as a treatment against Streptococcus iniae in tilapia (Oreochromis sp.). Aquaculture, 238, 97-105.

Ahmad, M.H. and Abdel-Tawwab, M. (2011) The use of caraway seed meal as a feed additive in fish diets: Growth performance, feed utilization, and whole-body composition of Nile tilapia, Oreochromis niloticus (L.) fingerlings. Aquaculture, 314, 110-114.

Ahmad, M.H., El Mesallamy, A.M.D., Samir, F. and Zahran, F. (2011) Effect of cinnamon (Cinnamomum zeylanicum) on growth performance, feed utilization, whole-body composition, and resistance to Aeromonas hydrophila in Nile tilapia. Journal of Applied Aquaculture, 23, 289-298. 
Alexander, C.P., Kirubakaran, C.J.W. and Michael, R.D. (2010) Water soluble fraction of Tinospora cordifolia leaves enhanced the non-specific immune mechanisms and disease resistance in Oreochromis mossambicus. Fish and Shellfish Immunology, 29, 765-772.

Ali, B.H., Blunden, G., Tanira, M.O. and Nemmar, A. (2008) Some phytochemical, pharmacological and toxicological properties of ginger (Zingiber officinale Roscoe): s review of recent research. Food and Chemical Toxicology, 46, 409-420.

Alishahi, M., Ranjbar, M.M., Ghorbanpour, M., Peyghan, R., Mesbah, M. and Razi-jalali, M. (2010) Effects of dietary Aloe vera on some specific and nonspecific immunity in the common carp (Cyprinus carpio). International Journal of Veterinary Research, 4, 189-195.

Allaby, M. (2012) A Dictionary of Plant Sciences. Oxford University Press, Oxford.

Aly, S.M. and Mohamed, M.F. (2010) Echinacea purpurea and Allium sativum as immunostimulants in fish culture using Nile tilapia (Oreochromis niloticus). Journal of Animal Physiology and Animal Nutrition, 94, e31-39.

Aly, S.M., Atti, N.M.A. and Mohamed, M.F. (2008) Effect of garlic on the survival, growth, resistance and quality of Oreochromis niloticus. Presented at the 8th International Symposium on Tilapia in Aquaculture, pp.277-296.

Anderson, D.P. (1992) Immunostimulants, adjuvants, and vaccine carriers in fish: applications to aquaculture. Annual Review of Fish Diseases, 2, 281-307.

Ardó, L., Yin, G., Xu, P., et al. (2008) Chinese herbs (Astragalus membranaceus and Lonicera japonica) and boron enhance the non-specific immune response of Nile tilapia (Oreochromis niloticus) and resistance against Aeromonas hydrophila. Aquaculture, 275, 26-33.

Ashley, P.J. (2007) Fish welfare: current issues in aquaculture. Applied Animal Behaviour Science, Fish Behavior and Welfare, 104, 199-235.

Ashraf, M.A. and Goda, S. (2008) Effect of dietary Ginseng herb (Ginsana@ G115) supplementation on growth, feed utilization, and hematological indices of Nile Tilapia, Oreochromis niloticus (L.), fingerlings. Journal of the World Aquaculture Society, 39, 205-214.

Awad, E. and Austin, B. (2010) Use of lupin, Lupinus perennis, mango, Mangifera indica, and stinging nettle, Urtica dioica, as feed additives to prevent Aeromonas hydrophila infection in rainbow trout, Oncorhynchus mykiss (Walbaum). Journal of Fish Diseases, 33, 413-420.

Awad, E., Mitchell, W.J. and Austin, B. (2011) Effect of dietary supplements on cytokine gene expression in rainbow trout, Oncorhynchus mykiss (Walbaum). Journal of Fish Diseases, 34, 629-634.

Ayyanar, M. and Ignacimuthu, S. (2011) Ethnobotanical survey of medicinal plants commonly used by Kani tribals in Tirunelveli hills of Western Ghats, India. Journal of Ethnopharmacology, 134, 851-864.

Balasubramanian, G., Sarathi, M., Kumar, S.R. and Hameed, A.S.S. (2007) Screening the antiviral activity of Indian medicinal plants against white spot syndrome virus in shrimp. Aquaculture, 263, 15-19.

Balasubramanian, G., Sarathi, M., Venkatesan, C., Thomas, J. and Sahul Hameed, A.S. (2008a) Oral administration of antiviral plant extract of Cynodon dactylon on a large scale production against White spot syndrome virus (WSSV) in Penaeus monodon. Aquaculture, 279, 2-5.

Balasubramanian, G., Sarathi, M., Venkatesan, C., Thomas, J. and Sahul Hameed, A.S. (2008b) Studies on the immunomodulatory effect of extract of Cynodon dactylon in shrimp, Penaeus monodon, and its efficacy to protect the shrimp from white spot syndrome virus (WSSV). Fish and Shellfish Immunology, 25, 820-828. 
Banskota, A.H., Tezuka, Y., Le Tran, Q. and Kadota, S. (2003) Chemical constituents and biological activities of Vietnamese medicinal plants. Current Topics in Medicinal Chemistry, 3, 227-248

Berman, B. (2012) New developments in the treatment of actinic keratosis: focus on ingenol mebutate gel. Clinical, Cosmetic and Investigational Dermatology, 5, 111-122.

Bhuvaneswari, R. and Balasundaram, C. (2006) Traditional Indian herbal extracts used in vitro against growth of the pathogenic bacteria - Aeromonas hydrophila. Israeli Journal of Aquaculture - Bamidgeh, 58, 89-96.

Bilen, S. and Bulut, M. (2010) Effects of laurel (Laurus nobilis) on the non-specific immune responses of rainbow trout (Oncorhynchus mykiss, Walbaum). Journal of Animal and Veterinary Advances, 9, 1275-1279.

Bilen, S., Bulut, M. and Bilen, A.M. (2011) Immunostimulant effects of Cotinus coggyria on rainbow trout (Oncorhynchus mykiss). Fish and Shellfish Immunology, 30, 451-455.

Bondad-Reantaso, M.G., Subasinghe, R.P., Arthur, J.R., et al. (2005) Disease and health management in Asian aquaculture. From Science to Solutions, plenary lectures presented at the 20th Conference of the World Association for the Advancement of Veterinary Parasitology, 132, 249-272.

Bower, A.M., Real Hernandez, L.M., Berhow, M.A. and de Mejia, E.G. (2014) Bioactive compounds from culinary herbs inhibit a molecular target for type 2 diabetes management, dipeptidyl peptidase IV. Journal of Agricultural and Food Chemistry, 62, 6147-6158.

Cabello, F.C. (2006) Heavy use of prophylactic antibiotics in aquaculture: a growing problem for human and animal health and for the environment. Environmental Microbiology, 8, 1137-1144.

Calixto, J.B. (2005) Twenty-five years of research on medicinal plants in Latin America: a personal view. Journal of Ethnopharmacology, 100, 131-134.

Carbonara, T., Pascale, R., Argentieri, M.P., et al. (2012) Phytochemical analysis of a herbal tea from Artemisia annua L. Journal of Pharmaceutical and Biomedical Analysis, 62, 79-86.

Caruana, S., Yoon, G.H., Freeman, M.A., Mackie, J.A. and Shinn, A.P. (2012) The efficacy of selected plant extracts and bioflavonoids in controlling infections of Saprolegnia australis (Saprolegniales; Oomycetes). Aquaculture, 358-359, 146-154.

Caruso, D., Lusiastuti, A.M., Taukhid, Slembrouck, J., Komarudin, O. and Legendre, M. (2013) Traditional pharmacopeia in small scale freshwater fish farms in West Java, Indonesia: an ethnoveterinary approach. Aquaculture, 416-417, 334-345.

Castro, S.B.R., Leal, C.A.G., Freire, F.R., Carvalho, D.A., Oliveira, D.F. and Figueiredo, H.C.P. (2008) Antibacterial activity of plant extracts from Brazil against fish pathogenic bacteria. Brazilian Journal of Microbiology, 39, 756-760.

Ćavar, S., Maksimović, M., Vidic, D. and Parić, A. (2012) Chemical composition and antioxidant and antimicrobial activity of essential oil of Artemisia annua L. from Bosnia. Industrial Crops and Products, 37, 479-485.

Chakrabarti, R., Srivastava, P.K., Verma, N. and Sharma, J. (2014) Effect of seeds of Achyranthes aspera on the immune responses and expression of some immune-related genes in carp Catla catla. Fish and Shellfish Immunology, 41, 64-69.

Chakraborty, S.B. and Hancz, C. (2011) Application of phytochemicals as immunostimulant, antipathogenic and antistress agents in finfish culture. Reviews in Aquaculture, 3, 103-119.

Chang, C.C., Tan, H.-C. and Cheng, W. (2013) Effects of dietary administration of water hyacinth (Eichhornia crassipes) extracts on the immune responses and disease resistance of giant freshwater prawn, Macrobrachium rosenbergii. Fish and Shellfish Immunology, 35, 92-100. 
Chaves, T.P., Santana, C.P., Véras, G., et al. (2013) Seasonal variation in the production of secondary metabolites and antimicrobial activity of two plant species used in Brazilian traditional medicine. African Journal of Biotechnology, 12, 847-853.

Chitmanat, C., Tongdonmuan, K., Khanom, P., Pachontis, P. and Nunsong, W. (2005) Antiparasitic, antibacterial and antifungal activities derived from a Terminalia catappa solution against some tilapia (Oreochromis niloticus) pathogens. Acta Horticulturae, 179-182.

Choudhury, S., Sree, A., Mukherjee, S.C., Pattnaik, P. and Bapuji, M. (2005) In vitro antibacterial activity of extracts of selected marine algae and mangroves against fish pathogens. Asian Fisheries Science, 18, 285-294.

Christybapita, D., Divyagnaneswari, M. and Michael, R.D. (2007) Oral administration of Eclipta alba leaf aqueous extract enhances the non-specific immune responses and disease resistance of Oreochromis mossambicus. Fish and Shellfish Immunology, 23, 840-852.

Citarasu, T. (2010) Herbal biomedicines: a new opportunity for aquaculture industry. Aquaculture International, 18, 403-414.

Citarasu, T., Sekar, R.R., Babu, M.M. and Marian, M.P. (2002) Developing Artemia enriched herbal diet for producing quality larvae in Penaeus monodon. Asian Fisheries Science, 15, 21-32.

Cox, S., Abu-Ghannam, N. and Gupta, S. (2010) An assessment of the antioxidant and antimicrobial activity of six species of edible Irish seaweeds. International Food Research Journal, 17, 205-220.

Dada, A.A. and Adeparusi, E.O. (2014) Dietary effects of two medicinal plants (Sesamum indicum) and (Croton zambesicus) on the reproductive indices in female African catfish (Clarias gariepinus) broodstock. Egyptian Journal of Aquatic Research, 38, 269-273.

Das, B.K., Pradhan, J. and Sahu, S. (2009) The effect of Euglena viridis on immune response of rohu, Labeo rohita (Ham.). Fish and Shellfish Immunology, 26, 871-876.

Davis, K.B., Griffin, B.R. and Gray, W.L. (2002) Effect of handling stress on susceptibility of channel catfish Ictalurus punctatus to Ichthyophthirius multifiliis and channel catfish virus infection. Aquaculture, 214, 55-66.

Dhayanithi, N.B., Kumar, T.T.A. and Balasubramanian, T. (2012) Effect of Excoecaria agallocha leaves against Aeromonas hydrophila in marine ornamental fish, Amphiprion sebae. Indian Journal of Geo-Marine Science, 41, 76-82.

Díaz-Rosales, P., Chabrillón, M., Abdala, R.T., Figueroa, F.L., Balebona, M.C. and Moriñigo, M.A. (2008) Effect of dietary administration of Porphyridium cruentum on the respiratory burst activity of sole, Solea senegalensis (Kaup), phagocytes. Journal of Fish Diseases, 31, 489-495.

Direkbusarakom, S. (2004) Application of medicinal herbs to aquaculture in Asia. Walailak Journal of Science and Technology, 1, 7-14.

Direkbusarakom, S., Herunsalee, A., Yoshimizu, M. and Ezura, Y. (1996) Antiviral activity of several Thai traditional herb extracts against fish pathogenic viruses. Fish Pathology, 31, 209-213.

Direkbusarakom, S., Ruangpan, L., Ezura, Y. and Yoshimizu, M. (1998) Protective efficacy of Clinacanthus nutans on yellow-head disease in black tiger shrimp (Penaeus monodon). Fish Pathology, 33, 401-404.

Divyagnaneswari, M., Christybapita, D. and Michael, R.D. (2007) Enhancement of nonspecific immunity and disease resistance in Oreochromis mossambicus by Solanum trilobatum leaf fractions. Fish and Shellish Immunology, 23, 249-259. 
Dorucu, M., Ozesen Colak, S., Ispir, U., Altinterim, B. and Celayir, Y. (2009) The effect of black cumin seeds, Nigella sativa, on the immune response of rainbow trout, Oncorhynchus mykiss. Mediterranean Aquaculture Journal, 2, 1-7.

Dubber, D. and Harder, T. (2008) Extracts of Ceramium rubrum, Mastocarpus stellatus and Laminaria digitata inhibit growth of marine fish pathogenic bacteria at ecologically realistic concentrations. Aquaculture, 274, 196-200.

Dugasani, S., Pichika, M.R., Nadarajah, V.D., Balijepalli, M.K., Tandra, S. and Korlakunta, J.N. (2010) Comparative antioxidant and anti-inflammatory effects of [6]-gingerol, [8]-gingerol, [10]-gingerol and [6]-shogaol. Journal of Ethnopharmacology, 127, 515-520.

Dügenci, S.K., Arda, N. and Candan, A. (2003) Some medicinal plants as immunostimulant for fish. Journal of Ethnopharmacology, 88, 99-106.

Efferth, T., Herrmann, F., Tahrani, A. and Wink, M. (2011) Cytotoxic activity of secondary metabolites derived from Artemisia annua L. towards cancer cells in comparison to its designated active constituent artemisinin. Phytomedicine, 18, 959-969.

Ekanem, A.P. and Brisibe, E.A. (2010) Effects of ethanol extract of Artemisia annua L. against monogenean parasites of Heterobranchus longifilis. Parasitology Research, 106, 1135-1139.

Ekanem, A.P., Obiekezie, A., Kloas, W. and Knopf, K. (2004) Effects of crude extracts of Mucuna pruriens (Fabaceae) and Carica papaya (Caricaceae) against the protozoan fish parasite Ichthyophthirius multifiliis. Parasitology Research, 92, 361-366.

Ekanem, A.P., Ekpo, I.A., Morah, F., Amanke, E. and Afangide, U. (2007) Acute toxicity of ethanol extracts from two ichthyotoxic plants Adenia cissampeloides (Passifloraceae) and Blighia sapida (Sapindaceae) to one week old Heterobranchus longifilis juveniles. Nigerian Journal of Botany, 20, 157-161.

El-Deen, A.I.E. and Mohamed, R.A. (2009) Application of some medicinal plants to eliminate Trichodina sp in tilapia (Oreochromis niloticus). Report and Opinion, 1, 1-5.

El-Ghorab, A.H., Nauman, M., Anjum, F.M., Hussain, S. and Nadeem, M. (2010) A comparative study on chemical composition and antioxidant activity of ginger (Zingiber officinale) and cumin (Cuminum cyminum). Journal of Agricultural and Food Chemistry, 58, 8231-8237.

Ernst, M., Grace, O.M., Saslis-Lagoudakis, C.H., Nilsson, N., Simonsen, H.T. and Rønsted, N. (2015) Global medicinal uses of Euphorbia L. (Euphorbiaceae). Journal of Ethnopharmacology, 176, 90-101.

Fang, S.H., Rao, Y.K. and Tzeng, Y.M. (2008) Anti-oxidant and inflammatory mediator's growth inhibitory effects of compounds isolated from Phyllanthus urinaria. Journal of Ethnopharmacology, 116, 333-340.

Ferreira, S., Santos, J., Duarte, A., Queiroz, J.A. and Domingues, F.C. (2012) Screening of antimicrobial activity of Cistus ladanifer and Arbutus unedo extracts. Natural Products Research, 26, 1557-1560.

Food and Agriculture Organization of the United Nations, Fisheries and Aquaculture Department (2014) The State of World Fisheries and Aquaculture. Food and Agriculture Organization of the United Nations, Rome.

Forwood, J.M., Harris, J.O. and Deveney, M.R. (2013) Efficacy of current and alternative bath treatments for Lepidotrema bidyana infecting silver perch, Bidyanus bidyanus. Aquaculture, 416-417, 65-71.

Francis, G., Makkar, H.P.S. and Becker, K. (2005) Quillaja saponins - a natural growth promoter for fish. Animal Feed Science and Technology, 121, 147-157.

Fu, Y.W., Hou, W.Y., Yeh, S.T., Li, C.H. and Chen, J.C. (2007) The immunostimulatory effects of hot-water extract of Gelidium amansii via immersion, injection and dietary administrations on 
white shrimp Litopenaeus vannamei and its resistance against Vibrio alginolyticus. Fish and Shellfish Immunology, 22, 673-685.

Fu, Y., Zhang, Q., Xu, D.H., et al. (2014) Parasiticidal effects of Morus alba root bark extracts against Ichthyophthirius multifiliis infecting grass carp. Diseases of Aquatic Organisms,108, 129-136.

Genovese, G., Faggio, C., Gugliandolo, C., et al. (2012) In vitro evaluation of antibacterial activity of Asparagopsis taxiformis from the Straits of Messina against pathogens relevant in aquaculture. Marine Environmental Research, 73, 1-6.

Genovese, G., Leitner, S., Minicante, S.A. and Lass-Flörl, C. (2013) The Mediterranean red alga Asparagopsis taxiformis has antifungal activity against Aspergillus species. Mycoses, 56, 516-519.

Gormez, O. and Diler, O. (2014) In vitro antifungal activity of essential oils from Tymbra, Origanum, Satureja species and some pure compounds on the fish pathogenic fungus, Saprolegnia parasitica. Aquaculture Research, 45, 1196-1201.

Gostner, J.M., Wrulich, O.A., Jenny, M., Fuchs, D. and Ueberall, F. (2012) An update on the strategies in multicomponent activity monitoring within the phytopharmaceutical field. $B M C$ Complementary and Alternative Medicine, 12, 18.

Haghighi, M. and Rohani, M.S. (2013) The effects of powdered ginger (Zingiber officinale) on the haematological and immunological parameters of rainbow trout Oncorhynchus mykiss. Journal of Medicinal Plant and Herbal Therapy Research, 1, 8-12.

Hao, B., Liu, G.L., Hu, X.G. and Wang, G.X. (2012) Bioassay-guided isolation and identification of active compounds from Semen pharbitidis against Dactylogyrus intermedius (Monogenea) in goldfish (Carassius auratus). Veterinary Parasitology, 187, 452-458.

Harikrishnan, R., Heo, J., Balasundaram, C., et al. (2010a) Effect of traditional Korean medicinal (TKM) triherbal extract on the innate immune system and disease resistance in Paralichthys olivaceus against Uronema marinum. Veterinary Parasitology, 170, 1-7.

Harikrishnan, R., Heo, J., Balasundaram, C., et al. (2010b) Effect of Punica granatum solvent extracts on immune system and disease resistance in Paralichthys olivaceus against lymphocystis disease virus (LDV). Fish and Shellfish Immunology, 29, 668-673.

Harikrishnan, R., Balasundaram, C., Kim, M.C., Kim, J.S., Han, Y.J. and Heo, M.S. (2010c) Effect of a mixed herb-enriched diet on the innate immune response and disease resistance of Paralichthys olivaceus against Philasterides dicentrarchi infection. Journal of Aquatic Animal Health, 22, 235-243.

Harikrishnan, R., Balasundaram, C. and Heo, M.S. (2011a) Impact of plant products on innate and adaptive immune system of cultured finfish and shellfish. Aquaculture, 317, 1-15.

Harikrishnan, R., Kim, J.S., Kim, M.C., Balasundaram, C. and Heo, M.S. (2011b) Kalopanax pictus as feed additive controls bacterial and parasitic infections in kelp grouper, Epinephelus bruneus. Fish and Shellfish Immunology, 31, 801-807.

Harikrishnan, R., Kim, J.S., Kim, M.C., Balasundaram, C. and Heo, M.S. (2011c) Lactuca indica extract as feed additive enhances immunological parameters and disease resistance in Epinephelus bruneus to Streptococcus iniae. Aquaculture, 318, 43-47.

Harikrishnan, R., Kim, J.S., Kim, M.C., Balasundaram, C. and Heo, M.S. (2011d) Styrax japonica supplementation diet enhances the innate immune response in Epinephelus bruneus against bacterial and protozoan infections. Experimental Parasitology, 129, 260-265.

Harikrishnan, R., Kim, M.C., Kim, J.S., Kim, D.H., Hong, S.H. and Heo, M.S. (2011e) Alnus firma supplementation diet on haematology and innate immune response in olive flounder against Tenacibaculum maritimum. Bulletin of the Veterinary Institute in Pulawy, 55, 649-655. 
Harikrishnan, R., Kim, J.S., Kim, M.C., Balasundaram, C. and Heo, M.S. (2011f) Prunella vulgaris enhances the non-specific immune response and disease resistance of Paralichthys olivaceus against Uronema marinum. Aquaculture, 318, 61-66.

Harikrishnan, R., Balasundaram, C. and Heo, M.S. (2012a) Effect of Inonotus obliquus enriched diet on hematology, immune response, and disease protection in kelp grouper, Epinephelus bruneus against Vibrio harveyi. Aquaculture, 344-349, 48-53.

Harikrishnan, R., Kim, D.H., Hong, S.H., Mariappan, P., Balasundaram, C. and Heo, M.S. (2012b) Non-specific immune response and disease resistance induced by Siegesbeckia glabrescens against Vibrio parahaemolyticus in Epinephelus bruneus. Fish and Shellfish Immunology, 33, 359-364.

Harikrishnan, R., Kim, J.S., Kim, M.C., et al. (2012c) Effect of dietary supplementation with Suaeda maritima on blood physiology, innate immune response, and disease resistance in olive flounder against Miamiensis avidus. Experimental Parasitology, 131, 195-203.

Hashemi, K.S.M., Sadeghpour, H.M. and Gholampour, A.I. (2012) Isolation of Saprolegnia and the influence of root ethanolic extract of Ruta graveolens on Saprolegnia. Spp growth. International Journal of Bioscience, Biochemistry and Bioinformatics, 2, 64-67.

Hecker, E. (1968) Cocarcinogenic principles from the seed oil of Croton tiglium and from other Euphorbiaceae. Cancer Research, 28, 2338-2348.

Hoareau, L. and DaSilva, E.J. (1999) Medicinal plants: a re-emerging health aid. Electronic Journal of Biotechnology, 2, 56-70.

Hoseinifar, S.H., Khalili, M., Rufchaei, R., et al. (2015) Effects of date palm fruit extracts on skin mucosal immunity, immune related genes expression and growth performance of common carp (Cyprinus carpio) fry. Fish and Shellfish Immunology, 47, 706-711.

Huang, A.G., Yi, Y.L., Ling, F., et al. (2013) Screening of plant extracts for anthelmintic activity against Dactylogyrus intermedius (Monogenea) in goldfish (Carassius auratus). Parasitology Research, 112, 4065-4072.

Huang, X., Zhou, H. and Zhang, H. (2006) The effect of Sargassum fusiforme polysaccharide extracts on vibriosis resistance and immune activity of the shrimp, Fenneropenaeus chinensis. Fish and Shellfish Immunology, 20, 750-757.

Huang, X.L., Liu, R.J., Whyte, S., et al. (2015) The in vitro antifungal activity of 30 Chinese herb extracts to Saprolegnia sp. Journal of Applied Ichtyology, 31, 681-686.

Hutson, K.S., Mata, L., Paul, N.A. and de Nys, R. (2012) Seaweed extracts as a natural control against the monogenean ectoparasite, Neobenedenia sp., infecting farmed barramundi (Lates calcarifer). International Journal of Parasitology, 42, 1135-1141.

Iguchi, K., Ogawa, K., Nagae, M. and Ito, F. (2003) The influence of rearing density on stress response and disease susceptibility of ayu (Plecoglossus altivelis). Aquaculture, 220, 515-523.

Innocent, X., Aly-Fathima, M.S. and Dhanalakshmi (2011) Studies on the immunostimulant activity of Coriandrum sativum and resistance to Aeromonas hydrophila in Catla catla. Journal of Applied Pharmaceutical Science, 1, 132-135.

Ji, J., Lu, C., Kang, Y., Wang, G.X. and Chen, P. (2012) Screening of 42 medicinal plants for in vivo anthelmintic activity against Dactylogyrus intermedius (Monogenea) in goldfish (Carassius auratus). Parasitology Research, 111, 97-104.

Ji, S.C., Jeong, G.S., Gwang-Soon, I., Lee, S.W., Yoo, J.H. and Takii, K. (2007a) Dietary medicinal herbs improve growth performance, fatty acid utilization, and stress recovery of Japanese flounder. Fisheries Science, 73, 70-76.

Ji, S.C., Takaoka, O., Jeong G.S., et al. (2007b) Dietary medicinal herbs improve growth and some non-specific immunity of red sea bream Pagrus major. Fisheries Science, 73, 63-69. 
Jiang, J. and Wu, Z. (2004) Influences of traditional Chinese medicine on non-specific immunity of Jian Carp (Cyprinus carpio var. Jian). Fish and Shellfish Immunology, 16, 185-191.

Jinish, L.J.G. (2002) Ayurvedic phytotherapeutics against Vibrio infections in grouper, Epinephelus tauvina, MSc dissertation, M.S. University, Tirunelveli, Tamil Nadu, India.

Juteau, F., Masotti, V., Bessière, J.M., Dherbomez, M. and Viano, J. (2002) Antibacterial and antioxidant activities of Artemisia annua essential oil. Fitoterapia, 73, 532-535.

Kaleeswaran, B., Ilavenil, S. and Ravikumar, S. (2011) Dietary supplementation with Cynodon dactylon (L.) enhances innate immunity and disease resistance of Indian major carp, Catla catla (Ham.). Fish and Shellfish Immunology, 31, 953-962.

Kaminskyy, V., Kulachkovskyy, O. and Stoika, R. (2008) A decisive role of mitochondria in defining rate and intensity of apoptosis induction by different alkaloids. Toxicology Letters, 177, $168-181$.

Kanani, H.G., Nobahar, Z., Kakoolaki, S. and Jafarian, H. (2014) Effect of ginger- and garlic-supplemented diet on growth performance, some hematological parameters and immune responses in juvenile Huso huso. Fish Physiology and Biochemistry, 40, 481-490.

Kanjana, K., Radtanatip, T., Asuvapongpatana, S., Withyachumnarnkul, B. and Wongprasert, K. (2011) Solvent extracts of the red seaweed Gracilaria fisheri prevent Vibrio harveyi infections in the black tiger shrimp Penaeus monodon. Fish and Shellfish Immunology, 30, 389-396.

Karagouni, E., Athanassopoulou, F., Lytra, A., Komis, C. and Dotsika, E. (2005) Antiparasitic and immunomodulatory effect of innovative treatments against Myxobolus $s p$. infection in Diplodus puntazzo. Veterinary Parasitology, 134, 215-228.

Kavitha, C., Ramesh, M., Kumaran, S.S. and Lakshmi, S.A. (2012) Toxicity of Moringa oleifera seed extract on some hematological and biochemical profiles in a freshwater fish, Cyprinus carpio. Experimental and Toxicologic Pathology, 64, 681-687.

Kemény-Beke, A., Aradi, J., Damjanovich, J., et al. (2006) Apoptotic response of uveal melanoma cells upon treatment with chelidonine, sanguinarine and chelerythrine. Cancer Letters, 237, $67-75$.

Khansari, A., Yavari, V., Alishahi, M., et al. (2013) Effects of Oliviera decumbens and Satureja khuzestanica extract on some immunological and haematological parameters of Cyprinus carpio. Comparative Clinical Pathology, 22, 339-342.

Khlifi, D., Hayouni, E.A., Valentin, A., et al. (2013) LC-MS analysis, anticancer, antioxidant and antimalarial activities of Cynodon dactylon L. extracts. Industrial Crops and Products, 45, 240-247.

Kim, J.S., Harikrishnan, R., Kim, M.C., et al. (2011) Enhancement of Eriobotrya japonica extracts on non-specific immune response and disease resistance in kelp grouper Epinephelus bruneus against Vibrio carchariae. Fish and Shellfish Immunology, 31, 1193-1200.

Kim, K.H., Hwang, Y.J. and Bai, S.C. (1999) Resistance to Vibrio alginolyticus in juvenile rockfish (Sebastes schlegeli) fed diets containing different doses of aloe. Aquaculture, 180, 13-21.

Kirubakaran, C.J.W., C.P. Alexander, and Michael, R.D. (2010) Enhancement of non-specific immune responses and disease resistance on oral administration of Nyctanthes arbortristis seed extract in Oreochromis mossambicus (Peters). Aquaculture Research, 41, 1630-1639.

Klayman, D.L. (1985) Qinghaosu (artemisinin): an antimalarial drug from China. Science, 228, 1049-1055.

Kolanjinathan, K., Ganesh, P. and Govindarajan, M. (2009) Antibacterial activity of ethanol extracts of seaweeds against fish bacterial pathogens. European Reviews in Medicine and Pharmacology Sciences, 13, 173-177. 
Krogdahl, A., Penn, M., Thorsen, J., Refstie, S. and Bakke, A.M. (2010) Important antinutrients in plant feedstuffs for aquaculture: an update on recent findings regarding responses in salmonids. Aquaculture Research, 41, 333-344.

Kumar, S., Raman, R.P., Pandey, P.K., Mohanty, S., Kumar, A. and Kumar, K. (2013) Effect of orally administered azadirachtin on non-specific immune parameters of goldfish Carassius auratus (Linn. 1758) and resistance against Aeromonas hydrophila. Fish and Shellfish Immunology, 34, 564-573.

Kumar, S., Chandra, P., Bajpai, V., et al. (2015) Rapid qualitative and quantitative analysis of bioactive compounds from Phyllanthus amarus using LC/MS/MS techniques. Industrial Crops and Products, 69, 143-152.

Kupchan, S.M., Uchida, I., Branfman, A.R., Dailey, R.G. and Fei, B.Y. (1976) Antileukemic principles isolated from euphorbiaceae plants. Science, 191, 571-572.

Lee, J.Y. and Gao, Y. (2012) Review of the application of garlic, Allium sativum, in aquaculture. Journal of the World Aquaculture Society, 43, 447-458.

Li, A., Sun, A. and Liu, R. (2005) Preparative isolation and purification of costunolide and dehydrocostuslactone from Aucklandia lappa Decne by high-speed counter-current chromatography. Journal of Chromatography A, 1076, 193-197.

Ling, F., Wang, J.G., Lu, C., Wang, G.X., Lui, Y.H. and Gong, X.N. (2012) Effects of aqueous extract of Capsicum frutescens (Solanaceae) against the fish ectoparasite Ichthyophthirius multifiliis. Parasitology Research, 111, 841-848.

Ling, F., Lu, C., Tu, X., et al. (2013) Antiprotozoal screening of traditional medicinal plants: evaluation of crude extract of Psoralea corylifolia against Ichthyophthirius multifiliis. Parasitology Research, 112, 2231-2240.

Liu, C., Tsai, W., Lin, Y., Liao, J., Chen, C. and Kuo, Y. (2004) The extracts from Nelumbo nucifera suppress cell cycle progression, cytokine genes expression, and cell proliferation in human peripheral blood mononuclear cells. Life Science, 75, 699-716.

Liu, G., Dong, J., Wang, H., Hashi, Y. and Chen, S. (2011) Characterization of alkaloids in Sophora flavescens Ait. by high-performance liquid chromatography-electrospray ionization tandem mass spectrometry. Journal of Pharmaceutical and Biomedical Analysis, 54, 1065-1072.

Logambal, S.M., Venkatalakshmi, S. and Michael, R.D. (2000) Immunostimulatory effect of leaf extract of Ocimum sanctum Linn. in Oreochromis mossambicus (Peters). Hydrobiologia, 430, 113-120.

Lu, C., Zhang, H.Y., Ji, J. and Wang, G.X. (2012) In vivo anthelmintic activity of Dryopteris crassirhizoma, Kochia scoparia, and Polygala tenuifolia against Dactylogyrus intermedius (Monogenea) in goldfish (Carassius auratus). Parasitology Research, 110, 1085-1090.

Mai, T., Tintillier, F., Lucasson, A., et al. (2015) Quorum sensing inhibitors from Leucette chagonensis Dendy, 1863. Letters in Applied Microbiology, 61, 311-317.

Malikova, J., Zdarilova, A. and Hlobilkova, A. (2006) Effects of sanguinarine and chelerythrine on the cell cycle and apoptosis. Biomedical Papers of the Medical Faculty of the University Palacky, Olomouc, Czech Republic, 150, 5-12.

Malmir, M., Gohari, A.R., Saeidnia, S. and Silva, O. (2015) A new bioactive monoterpene-flavonoid from Satureja khuzistanica. Fitoterapia, 105, 107-112.

Manilal, A., Selvin, J., Kiran, G. K., et al. (2009) Antimicrobial potential and seasonality of red algae collected from the southwest coast of India tested against shrimp, human and phytopathogens. Annals of Microbiology, 59, 207-219. 
Manilal, A., Sujith, S., Sabarathnam, B., et al. (2010) Bioactivity of the red algae Asparagopsis taxiformis collected from the southwestern coast of India. Brazilian Journal of Oceanography, 58, 93-100.

Manilal, A., Selvin, J. and Georges, S. (2012) In vivo therapeutic potentiality of red seaweed, Asparagopsis (Bonnemaisoniales, Rhodophyta) in the treatment of Vibriosis in Penaeus monodon Fabricius. Saudi Journal of Biological Science, 19, 165-175.

Manilal, A., Selvin, J. and Sugathan, S. (2013) Immuno-modulatory efficacy of Indian red algae, Asparagopsis taxiformis, in Penaeus monodon. Journal of Applied Aquaculture, 25, 81-93.

Martins, M.L., Moraes, F.R., Miyazaki, D.M.Y., et al. (2002) Alternative treatment for Anacanthorus penilabiatus (Monogenea: Dactylogyridae) infection in cultivated pacu, Piaractus mesopotamicus (Osteichthyes: Characidae) in Brazil and its haematological effects. Parasite, 9, 175-180.

Micol, V., Caturla, N., Perez-Fons, L., Mas, V., Perez, L. and Estepa, A. (2005) The olive leaf extract exhibits antiviral activity against viral haemorrhagic septicaemia rhabdovirus (VHSV). Antiviral Research, 66, 129-136.

Militz, T.A., Southgate, P.C., Carton, A.G. and Hutson, K.S. (2013) Dietary supplementation of garlic (Allium sativum) to prevent monogenean infection in aquaculture. Aquaculture, 408-409, 95-99.

Millet, C.O.M., Lloyd, D., Williams, C., et al. (2011) Effect of garlic and allium-derived products on the growth and metabolism of Spironucleus vortens. Experimental Parasitology, 127, 490-499.

Mimica-Dukic, N. and Bozin, B. (2008) Mentha L. species (Lamiaceae) as promising sources of bioactive secondary metabolites. Current Pharmaceutical Design, 14, 3141-3150.

Minomol, M. (2005) Culture of Gold fish Carassius auratus using medicinal plants having immunostimulant characteristics. MPhil dissertation, MS University, Tamil Nadu, India.

Miranda, C.D. and Zemelman, R. (2002) Antimicrobial multiresistance in bacteria isolated from freshwater Chilean salmon farms. Science of the Total Environment, 293, 207-218.

Mishra, L.C., Singh, B.B. and Dagenais, S. (2000) Scientific basis for the therapeutic use of Withania somnifera (ashwagandha): a review. Alternative Medicine Review, 5, 334-346.

Molares, S. and Ladio, A. (2011) The usefulness of edible and medicinal Fabaceae in Argentine and Chilean Patagonia: environmental availability and other sources of supply. Evidence-Based Complementary and Alternative Medicine, 2012, e901918.

Neves, J.M., Matos, C., Moutinho, C., Queiroz, G. and Gomes, L.R. (2009) Ethnopharmacological notes about ancient uses of medicinal plants in Trás-os-Montes (northern of Portugal). Journal of Ethnopharmacology, 124, 270-283.

Nya, E.J. and Austin, B. (2009) Use of dietary ginger, Zingiber officinale Roscoe, as an immunostimulant to control Aeromonas hydrophila infections in rainbow trout, Oncorhynchus mykiss (Walbaum). Journal of Fish Diseases, 32, 971-977.

Nya, E.J., Dawood, Z. and Austin, B. (2010) The garlic component, allicin, prevents disease caused by Aeromonas hydrophila in rainbow trout, Oncorhynchus mykiss (Walbaum). Journal of Fish Diseases, 33, 293-300.

Okeke, M.I., Iroegbu, C.U., Jideofor, C.O., Okoli, A. and Esimone, C.O. (2001) Antimicrobial activity of ethanol extracts of two indigenous Nigerian spices. Journal of Herbs, Spices and Medicinal Plants, 8, 39-48.

Oladosu-Ayayi, R.N., George, F.O.A., Obasa, S.O. and Bankole, M.O. (2013) Effects of some plant extracts on some biological parameters of catfish Clarias gariepinus (Burchell, 1822). Journal of Fisheries and Aquatic Science, 8, 142-147. 
Olusola, S.E., Emikpe, B.O. and Olaifa, F.E. (2013) The potentials of medicinal plants extracts as bio-antimicrobial in aquaculture. International Journal of Medicinal Aromatic Plants, 3, 404-412.

Pachanawan, A., Phumkhachorn, P. and Rattanachaikunsopon, P. (2008) Potential of Psidium guajava supplemented fish diets in controlling Aeromonas hydrophila infection in tilapia (Oreochromis niloticus). Journal of Bioscience and Bioengineering, 5, 419-424.

Pakravan, S., Hajimoradloo, A. and Ghorbani, R. (2012) Effect of dietary willow herb, Epilobium hirsutum extract on growth performance, body composition, haematological parameters and Aeromonas hydrophila challenge on common carp, Cyprinus carpio. Aquaculture Research, 43, 861-869.

Pan, T.S., Yan, M.C., Chen, S.B. and Wang, X.P. (2013) Effects of ten traditional chinese herbs on immune response and disease resistance of Sciaenops ocellatus (Actinopterygii: Perciformes: Sciaenidae). Acta Ichthyologica Piscatoria, 43, 41-49.

Park, K.H. and Choi, S.H. (2012) The effect of mistletoe, Viscum album coloratum, extract on innate immune response of Nile tilapia (Oreochromis niloticus). Fish and Shellfish Immunology, 32, 1016-1021.

Pasnik, D.J., Evans, J.J., Panangala, V.S., Klesius, P.H., Shelby, R.A. and Shoemaker, C.A. (2005) Antigenicity of Streptococcus agalactiae extracellular products and vaccine efficacy. Journal of Fish Diseases, 28, 205-212.

Pattanayak, P., Behera, P., Das, D. and Panda, S.K. (2010) Ocimum sanctum Linn. A reservoir plant for therapeutic applications: an overview. Pharmacognosy Review, 4, 95-105.

Pavaraj, M., Balasubram, V., Baskaran, S. and Ramasamy, P. (2011) Development of immunity by extract of medicinal plant Ocimum sanctum on common carp Cyprinus carpio (L.). Research Journal of Immunology, 4, 12-18.

Petrovska, B.B. (2012) Historical review of medicinal plants' usage. Pharmacognosy Review, 6, $1-5$.

Philchenkov, A., Kaminskyy, V., Zavelevich, M. and Stoika, R. (2008) Apoptogenic activity of two benzophenanthridine alkaloids from Chelidonium majus L. does not correlate with their DNA damaging effects. Toxicology In Vitro, 22, 287-295.

Pirbalouti, G., Broujeni, N., Momeni, M., Poor, M. and Hamedi, B. (2011) Antibacterial activity of Iranian medicinal plants against Streptococcus iniae isolated from rainbow trout (Oncorhynchus mykiss). Archives of Biological Science, 63, 59-66.

Pratheepa, V. and Sukumaran, N. (2011) Specific and nonspecific immunostimulation study of Euphorbia hirta on Pseudomonas fluorescens-infected Cyprinus carpio. Pharmaceutical Biology, 48, 484-491.

Pratheepa, V., Ramesh, S. and Sukumaran, N. (2010) Immunomodulatory effect of Aegle marmelos leaf extract on freshwater fish Cyprinus carpio infected by bacterial pathogen Aeromonas hydrophila. Pharmaceutical Biology, 48, 1224-123.

Punitha, S.M.J., Babu, M.M., Sivaram, V., et al. (2008) Immunostimulating influence of herbal biomedicines on nonspecific immunity in Grouper Epinephelus tauvina juvenile against Vibrio harveyi infection. Aquaculture International, 16, 511-523.

Purivirojkul, W. (2012) Potential application of extracts from Indian almond (Terminalia catappa Linn.) leaves in Siamese fighting fish (Betta splendens Regan) culture. Communications in Agricultural Applied Biological Sciences, 77, 439-448.

Putra, A.A.S. Santoso, U., Lee, M.C. and Nan, F.H. (2013) Effects of dietary katuk leaf extract on growth performance, feeding behavior and water quality of grouper Epinephelus coioides. Aceh International Journal of Science and Technology, 2. 
Qi, W.Y., Zhang, W.Y., Shen, Y., Leng, Y., Gao, K. and Yue, J.M. (2014) Ingol-type diterpenes from Euphorbia antiquorum with mouse $11 \beta$-hydroxysteroid dehydrogenase type 1 inhibition activity. Journal of Natural Products, 77, 1452-1458.

Radhakrishnan, S., Bhavan, P.S., Seenivasan, C., Shanthi, R. and Poongodi, R. (2014) Influence of medicinal herbs (Alteranthera sessilis, Eclipta alba and Cissus quadrangularis) on growth and biochemical parameters of the freshwater prawn Macrobrachium rosenbergii. Aquaculture International, 22, 551-572.

Raja Rajeswari, P., Velmurugan, S., Babu, M.M., Dhas, S.A., Kesavan, K. and Citarasu, T. (2012) A study on the influence of selected Indian herbal active principles on enhancing the immune system in Fenneropenaeus indicus against Vibrio harveyi infection. Aquaculture International, 20, 1009-1020.

Rajendiran, A., Natarjan, E. and Subramanian, P. (2008) Control of Aeromonas hydrophila infection in spotted snakehead Channa punctatus, by Solanum nigrum L., a medicinal plant. Journal of the World Aquaculture Society, 39, 375-383.

Rajeswari, P.R., Velmurugan, S., Babu, M.M., Dhas, S.A., Kesavan, K. and Citarasu, T. (2012) A study on the influence of selected Indian herbal active principles on enhancing the immune system in Fenneropenaeus indicus against Vibrio harveyi infection. Aquaculture International, 20, 1009-1020.

Rattanachaikunsopon, P. and Phumkhachorn, P. (2009a) Potential of Chinese chive oil as a natural antimicrobial for controlling Flavobacterium columnare infection in Nile tilapia Oreochromis niloticus. Fisheries Science, 75, 1431.

Rattanachaikunsopon, P. and Phumkhachorn, P. (2009b) Prophylactic effect of Andrographis paniculata extracts against Streptococcus agalactiae infection in Nile tilapia (Oreochromis niloticus). Journal of Bioscience and Bioengineering, 107, 579-582.

Rattanachaikunsopon, P. and Phumkhachorn, P. (2010a) Assessment of synergistic efficacy of carvacrol and cymene against Edwardsiella tarda in vitro and in Tilapia (Oreochromis niloticus). African Journal of Microbiology Research, 4, 420-425.

Rattanachaikunsopon, P. and Phumkhachorn, P. (2010b) Effect of Cratoxylum formosum on innate immune response and disease resistance against Streptococcus agalactiae in tilapia Oreochromis niloticus. Fisheries Science, 76, 653-659.

Reverter, M., Bontemps, N., Lecchini, D., Banaigs, B. and Sasal, P. (2014) Use of plant extracts in fish aquaculture as an alternative to chemotherapy: current status and future perspectives. Aquaculture, 433, 50-61.

Rico, A., Phu, T.M., Satapornvanit, K., et al. (2013) Use of veterinary medicines, feed additives and probiotics in four major internationally traded aquaculture species farmed in Asia. Aquaculture, 412-413, 231-243.

Romero Ormazábal, J.M., Feijoó, C.G. and Navarrete Wallace, P.A. (2012) Antibiotics in aquacul ture - use, abuse and alternatives, in Health and Environment in Aquaculture (eds E.D. Carvalho, J.S. David and R.J. Silva), InTech, Croatia, p. 159.

Roomiani, L., Soltani, M., Akhondzadeh Basti, A., Mahmoodi, A., Taheri , Mirghaed, A. and Yadollahi, F. (2013) Evaluation of the chemical composition and in vitro antimicrobial activity of Rosmarinus officinalis, Zataria multiflora, Anethum graveolens and Eucalyptus globulus against Streptococcus iniae; the cause of zoonotic disease in farmed fish. Iranian Journal of Fish Science, 12, 702-716.

Sahu, S., Das, B.K., Pradhan, J., Mohapatra, B.C., Mishra, B.K. and Sarangi, N. (2007) Effect of Mangifera indica kernel as a feed additive on immunity and resistance to Aeromonas hydrophila in Labeo rohita in fingerlings. Fish and Shellfish Immunology, 23, 109-118. 
Sahu, S., Das, B.K., Mishra, B.K. and Pradhan, J. (2008) Effect of dietary Curcuma longa on enzymatic and immunological profiles of rohu, Labeo rohita ( Ham.), infected with Aeromonas hydrophila. Aquaculture Research, 39, 1720-1730.

Sakai, M. (1999) Current research status of fish immunostimulants. Aquaculture, 172, 63-92. Sambasivam, S., Karpagam, G., Chandran, R. and Khan, S.A. (2003) Toxicity of leaf extract of yellow oleander Thevetia nerifolia on Tilapia. Journal of Environmental Biology, 24, 201-204.

Schütz, K., Carle, R. and Schieber, A. (2006) Taraxacum - a review on its phytochemical and pharmacological profile. Journal of Ethnopharmacology, 107, 313-323.

Seo, C.S. and Shin, H.K. (2015) Simultaneous determination of three sesquiterpene lactones in Aucklandia lappa Decne by high-performance liquid chromatography. Pharmacognosy Magazine, 11, 562-566.

Seyfried, E.E., Newton, R.J., Iv, K.F.R., Pedersen, J.A. and McMahon, K.D. (2010) Occurrence of tetracycline resistance genes in aquaculture facilities with varying use of oxytetracycline. Microbial Ecology, 59, 799-807.

Shangliang, T., Hetrick, F.M., Roberson, B.S. and Baya, A. (1990) The antibacterial and antiviral activity of herbal extracts for fish pathogens. Journal of Ocean University of Qingdao, 02.

Shao, B., Xu, W., Dai, H., Tu, P., Li, Z. and Gao, X. (2004) A study on the immune receptors for polysaccharides from the roots of Astragalus membranaceus, a Chinese medicinal herb. Biochemical and Biophysial Research Communications, 320, 1103-1111.

Sharma, A., Deo, A.D., Tandel Riteshkumar, S., Chanu, T.I. and Das, A. (2010) Effect of Withania somnifera (L. Dunal) root as a feed additive on immunological parameters and disease resistance to Aeromonas hydrophila in Labeo rohita (Hamilton) fingerlings. Fish and Shellfish Immunology, 29, 508-512.

Sheikhzadeh, N., Nofouzi, K., Delazar, A. and Oushani, A.K. (2011) Immunomodulatory effects of decaffeinated green tea (Camellia sinensis) on the immune system of rainbow trout (Oncorhynchus mykiss). Fish and Shellfish Immunology, 31, 1268-1269.

Sirirustananun, N., Chen, J.C., Lin, Y.C., et al. (2011) Dietary administration of a Gracilaria tenuistipitata extract enhances the immune response and resistance against Vibrio alginolyticus and white spot syndrome virus in the white shrimp Litopenaeus vannamei. Fish and Shellfish Immunology, 31, 848-855.

Sivaram, V., Babu, M.M., Immanuel, G., Murugadass, S., Citarasu, T. and Marian, M. (2004) Growth and immune response of juvenil greasy groupers (Epinephelus tauvina) fed with herbal antibacterial active principle supplemented diets against Vibrio harveyi infections. Aquaculture, 237, 9-20.

Sivasankar, L., Santhiya, A.V. and Kanaga, V. (2015) A review on plants and herbal extracts against viral diseases in aquaculture. Journal of Medicinal Plants Studies, 3, 75-79.

Slaninová, I., Táborská, E., Bochoráková, H. and Slanina, J. (2001) Interaction of benzo[c]phenanthridine and protoberberine alkaloids with animal and yeast cells. Cell Biology and Toxicology, 17, 51-63.

Soltani, M., Sheikhzadeh, N., Ebrahimzadeh-Mousavi, H.A. and Zargar, A. (2010) Effects of Zataria multiflora essential oil on innate immune responses of common carp (Cyprinus carpio). Journal of Fisheries and Aquatic Science, 5, 191-199.

Srivastava, J., Chandra, H., Nautiyal, A.R. and Kalra, S.J.S. (2014) Antimicrobial resistance (AMR) and plant-derived antimicrobials (PDAms) as an alternative drug line to control infections. 3 Biotech, 4, 451-460. 
Sudhakaran, D.S., Srirekha, P., Devasree, L.D., Premsingh, S. and Michael, R.D. (2006) Immunostimulatory effect of Tinospora cordifolia Miers leaf extract in Oreochromis mossambicus. Indian Journal of Experimental Biology, 44, 726-732.

Sukoso, Nursyam, H., Sy, S.R. and Sormin, R.B.D. (2012) Study antibacterial and cytotoxic activities of seaweed Porphyra sp. extract. International Journal of Current Research, 4, $141-143$.

Syahidah, A., Saad, C.R., Daud, H.M. and Abdelhadi, Y.M. (2015) Status and potential of herbal applications in aquaculture: a review. Iranian Journal of Fisheries Sciences, 14, 27-44.

Takaoka, O., Ji, S.C., Ishimaru, K., et al. (2011) Effect of rotifer enrichment with herbal extracts on growth and resistance of red sea bream, Pagrus major (Temminck \& Schlegel) larvae against Vibrio anguillarum. Aquaculture Research, 42, 1824-1829.

Talpur, A.D., Ikhwanuddin, M. and Ambok Bolong, A.M. (2013) Nutritional effects of ginger (Zingiber officinale Roscoe) on immune response of Asian sea bass, Lates calcarifer (Bloch) and disease resistance against Vibrio harveyi. Aquaculture, 400-401, 46-52.

Tanekhy, M. and Fall, J. (2015) Expression of innate immunity genes in kuruma shrimp Marsupenaeus japonicus after in vivo stimulation with garlic extract (allicin). Veterinární Medicína, 60, 39-47.

Tang, W. and Eisenbrand, G. (1992) Chinese Drugs of Plant Origin, Springer Verlag, Berlin. Trinh, Q. and Le, L. (2014) An investigation of antidiabetic activities of bioactive compounds in Euphorbia hirta Linn using molecular docking and pharmacophore. Medicinal Chemistry Research, 23, 2033-2045.

Tu, X., Ling, F., Huang, A., Zhang, Q. and Wang, G. (2013) Anthelmintic efficacy of Santalum album (Santalaceae) against monogenean infections in goldfish. Parasitology Research, 112, 2839-2845.

Umeda, N., Nibe, H., Hara, T. and Hirazawa, N. (2006) Effects of various treatments on hatching of eggs and viability of oncomiracidia of the monogenean Pseudodactylogyrus anguillae and Pseudodactylogyrus bini. Aquaculture, 253, 148-153.

Van Hai, N. (2015) The use of medicinal plants as immunostimulants in aquaculture: a review. Aquaculture, 446, 88-96.

Vasudeva Rao, Y., Das, B.K., Jyotyrmayee, P. and Chakrabarti, R. (2006) Effect of Achyranthes aspera on the immunity and survival of Labeo rohita infected with Aeromonas hydrophila. Fish and Shellfish Immunology, 20, 263-273.

Walterová, D., Ulrichová, J., Válka, I., et al. (1995) Benzo[c]phenanthridine alkaloids sanguinarine and chelerythrine: biological activities and dental care applications. Biomedical Papers of the Medical Faculty of the University Palacky, Olomouc, Czech Republic, 139, 7-16.

Wang, G.X., Han, J., Zhao, L.W., Jiang, D.X., Liu, Y.T. and Liu, X.L. (2010a) Anthelmintic activity of steroidal saponins from Paris polyphylla. Phytomedicine, 17, 1102-1105.

Wang, G.X., Zhou, Z., Jiang, D.X., et al. (2010b) In vivo anthelmintic activity of five alkaloids from Macleaya microcarpa (Maxim) Fedde against Dactylogyrus intermedius in Carassius auratus. Veterinary Parasitology, 171, 305-313.

Wang, Q.K., Chen, C.X., Guo, Y.J., et al. (2011) Dietary polysaccharide from Angelica sinensis enhanced cellular defence responses and disease resistance of grouper Epinephelus malabaricus. Aquaculture International, 19, 945-956.

Wang, Y., Wu, Z.F., Wang, G.X., et al. (2011) In vivo anthelmintic activity of bruceine A and bruceine D from Brucea javanica against Dactylogyrus intermedius (Monogenea) in goldfish (Carassius auratus). Veterinary Parasitology, 177, 127-133. 
Wei, L.S., Musa, N., Sengm, C.T., Wee, W. and Shazili, N.A.M. (2008) Antimicrobial properties of tropical plants against 12 pathogenic bacteria isolated from aquatic organisms. African Journal of Biotechnology, 7, 2275-2278.

Wei, W.X., Pan, Y.J., Zhang, H., Lin, C.W. and Wei, T.Y. (2004) Two new compounds from Phyllanthus niruri. Chemistry of Natural Compounds, 40, 460-464.

Whittington, I.D. (2012) Benedenia seriolae and Neobenedenia species, in Fish Parasites: Pathobiology and Protection (eds P.T.K. Woo and K. Buchmann), CABI, Wallingford, pp. 225-244.

Won, K.M., Kim, P.K., Lee, S.H. and Park, S.I (2008) Effect of the residuum extract of Siberian ginseng Eleutherococcus senticosus on non-specific immunity in olive flounder Paralichthys olivaceus. Fisheries Science, 74, 635-641.

World Health Organization (2006) WHO Briefing on Malaria Treatment Guidelines and Artemisinin Monotherapies, WHO, Geneva.

Wu, C.C., Liu, C.H., Chang, Y.P. and Hsieh, S.L. (2010) Effects of hot-water extract of Toona sinensis on immune response and resistance to Aeromonas hydrophila in Oreochromis mossambicus. Fish and Shellfish Immunology, 29, 258-263.

Wu, Y.R., Gong, Q.F., Fang, H., Liang, W.W., Chen, M. and He, R.J. (2013) Effect of Sophora flavescens on non-specific immune response of tilapia (GIFT Oreochromis niloticus) and disease resistance against Streptococcus agalactiae. Fish and Shellfish Immunology, 34, $220-227$.

Wu, Z.F., Zhu, B., Wang, Y., Lu, C. and Wang, G.X. (2011) In vivo evaluation of anthelmintic potential of medicinal plant extracts against Dactylogyrus intermedius (Monogenea) in goldfish (Carassius auratus). Parasitology Research, 108, 1557-1563.

Xie, J., Liu, B., Zhou, Q., et al. (2008) Effects of anthraquinone extract from rhubarb Rheum officinale Bail on the crowding stress response and growth of common carp Cyprinus carpio var. Jian. Aquaculture, 281, 5-11.

Xue-Gang, H., Lei, L., Cheng, C., Kun, H., Xian-Le, Y. and Gao-Xue, W. (2013) In vitro screening of Chinese medicinal plants for antifungal activity against Saprolegnia sp. and Achlya klebsiana. North American Journal of Aquaculture, 75, 468-473.

Yao, J.Y., Zhou, Z.M., Pan, X., et al. (2011) In vivo anthelmintic activity of chelidonine from Chelidonium majus L. against Dactylogyrus intermedius in Carassius auratus. Parasitology Research, 109, 1465-1469.

Yeh, S.T., Lee, C.S. and Chen, J.C. (2006) Administration of hot-water extract of brown seaweed Sargassum duplicatum via immersion and injection enhances the immune resistance of white shrimp Litopenaeus vannamei. Fish and Shellfish Immunology, 20, 332-345.

Yi, Y.L., Lu, C., Hu, X.G., Ling, F. and Wang, G.X. (2012) Antiprotozoal activity of medicinal plants against Ichthyophthirius multifiliis in goldfish (Carassius auratus). Parasitology Research, 111, 1771-1778.

Yogeeswaran, A., Velmurugan, S., Punitha, S.M.J., et al. (2012) Protection of Penaeus monodon against white spot syndrome virus by inactivated vaccine with herbal immunostimulants. Fish and Shellfish Immunology, 32, 1058-1067.

Yoshida, T., Kruger, R. and Inglis, V. (1995) Augmentation of non-specific protection in African catfish, Clarias gariepinus (Burchell), by the long-term oral administration of immunostimulants. Journal of Fish Diseases, 18, 195-198.

Yuan, C., Li, D., Chen, W., et al. (2007) Administration of a herbal immunoregulation mixture enhances some immune parameters in carp (Cyprinus carpio). Fish Physiology and Biochemistry, 33, 93-101. 
Zbakh, H., Chiheb, H., Bouziane, H., Sánchez, V.M. and Riadi, H. (2012) Antibacterial activity of benthic marine algae extracts from the mediterranean coast of Morocco. Journal of Microbiology, Biotechnology and Food Science, 2, 219-228.

Zhang, J., Hu, X., Gao, W., et al. (2014) Pharmacokinetic study on costunolide and dehydrocostuslactone after oral administration of traditional medicine Aucklandia lappa Decne. by LC/MS/MS. Journal of Ethnopharmacology, 151, 191-197.

Zheng, Z.I., Tan, J.Y.W., Liu, H.Y., Zhou, X.H., Xiang, X. and Wang K.Y. (2009) Evaluation of oregano essential oil (Origanum heracleoticum L.) on growth, antioxidant effect and resistance against Aeromonas hydrophila in channel catfish (Ictalurus punctatus). Aquaculture, 292, 214-218. 
